TRANSACTIONS OF THE

AMERICAN MATHEMATICAL SOCIETY

Volume 355, Number 7, Pages 2857-2883

S 0002-9947(03)03248-3

Article electronically published on March 19, 2003

\title{
DISTRIBUTIONS OF CORANK 1 AND THEIR CHARACTERISTIC VECTOR FIELDS
}

\author{
B. JAKUBCZYK AND M. ZHITOMIRSKII
}

\begin{abstract}
We prove that any 1-parameter family of corank 1 distributions (or Pfaff equations) on a compact manifold $M^{n}$ is trivializable, i.e., transformable to a constant family by a family of diffeomorphisms, if all distributions of the family have the same characteristic line field. The characteristic line field is a field of tangent lines which is invariantly assigned to a corank one distribution. It is defined on $M^{n}$, if $n=2 k$, or on a subset of $M^{n}$ called the Martinet hypersurface, if $n=2 k+1$. Our second main result states that if two corank one distributions have the same characteristic line field and are close to each other, then they are equivalent via a diffeomorphism. This holds under a weak assumption on the singularities of the distributions. The second result implies that the abnormal curves of a distribution determine the equivalence class of the distribution, among distributions close to a given one.
\end{abstract}

\section{INTRODUCTION}

The well-known Gray theorem $G$ states that any 1-parameter family of contact structures on a compact manifold $M^{2 k+1}$ is trivializable, i.e., transformable to a constant family by a family of diffeomorphisms. Our first main result generalizes this theorem to the case of "singular contact structures", for which the contact condition is satisfied on a dense subset of $M$, and to corank one distributions on manifolds of even dimension. In these cases the family of distributions has to preserve, when the parameter changes, a characteristic line field. The characteristic line field is a field of tangent lines which is invariantly assigned to a corank one distribution (it is defined on $M^{n}$ if $n=2 k$, or on a hypersurface of $M^{n}$ if $n=2 k+1$ ).

Our second main result states that if two corank one distributions have the same characteristic line field and are close to each other, then they are equivalent via a diffeomorphism. It means, in particular, that the characteristic line field contains complete information about the geometry of singularities of the distribution. Our results hold under a weak assumption on the distributions, called condition (A), saying that the depth of a characteristic ideal of the distribution is nondegenerate at singular points of the characteristic line field.

Received by the editors January 9, 2002 and, in revised form, September 4, 2002.

2000 Mathematics Subject Classification. Primary 58A17; Secondary 53B99.

Key words and phrases. Pfaff equation, equivalence, contact structure, quasi-contact structure, singularity, invariants, line field, homotopy method.

The first author was supported by the Committee for Scientific Research (KBN), Poland, grant 2P03A 03516.

The second author was supported by the Fund for the Promotion of Research at the Technion. 
Let us state our second result in the simple case where the characteristic line field does not have singularities. Assume $n=2 k \geq 4$ and consider a smooth differential 1 -form $\omega$ on $M^{n}$. Let $\left(\omega \wedge(d \omega)^{k-1}\right)(p) \neq 0$ for all $p \in M^{n}$. Such a form $\omega$ defines the distribution $\Delta=\operatorname{ker} \omega$, called the quasi-contact structure defined by $\omega$. Then $d \omega(p)$, restricted to the distribution $\Delta(p)=\operatorname{ker} \omega(p)$, is of maximal possible rank $2 k-2$ and has 1-dimensional kernel. We define the characteristic line at $p$ by

$$
L_{p}=\left.\operatorname{ker} d \omega(p)\right|_{\Delta(p)} .
$$

The characteristic line field $L=L(\omega)$ is the field of characteristic lines $p \rightarrow L_{p}$ on $M$. The following fact is a special case of our Theorem 1.2, the case where singularities are absent. (It is also a special case of a theorem in [MZh], Appendix A, concerning corank 1 distributions of constant class.)

Theorem 0.1. Let $\Delta$ be the quasi-contact structure on a compact orientable manifold $M^{2 k}$ defined by a 1-form $\omega$, and let $\tilde{\omega}$ be a 1-form such that $L(\tilde{\omega})=L(\omega)$. If $\tilde{\omega}$ is sufficiently $C^{2}$-close to $\omega$, then there exists a diffeomorphism of $M^{2 k}$ sending $\tilde{\Delta}=\operatorname{ker} \tilde{\omega}$ to $\Delta$.

If a compact orientable manifold $M^{2 k}$ admits a quasi-contact structure defined by a global 1-form $\omega$, then its characteristic line field is generated by a nonvanishing global vector field and the Euler characteristic of $M^{2 k}$ is equal to zero. Thus a manifold $M^{2 k}$ with nonzero Euler characteristic admits only corank one distributions with singular characteristic line field. Even if $M^{2 k}$ admits a quasi-contact structure, singularities may appear naturally when restricting a corank one distribution to a submanifold of even dimension. Therefore, it is natural to ask whether Theorem 0.1 holds in the presence of singularities. Theorem 1.2 in Section 1 gives a positive answer under assumption (A), saying that the singularities of the characteristic vector field have a natural depth (and codimension, in the analytic case). This assumption excludes singularities of infinite codimension.

To state a similar result in the case $n=2 k+1$, we introduce the set of points where $\omega$ does not satisfy the contact condition:

$$
S=\left\{p \in M^{2 k+1}:\left(\omega \wedge(d \omega)^{k}\right)(p)=0\right\} .
$$

This set is called the Martinet hypersurface. The Martinet hypersurface is the set of zeros of the function

$$
H=\omega \wedge(d \omega)^{k} / \Omega,
$$

where $\Omega$ is a volume form. If $S$ is empty, i.e., $\omega$ is a contact 1 -form on $M^{2 k+1}$, then $\Delta=\operatorname{ker} \omega$ is globally equivalent to any distribution $\tilde{\Delta}$ sufficiently close to $\Delta$. This follows from the theorem of Gray mentioned above. Assume now that $S$ is nonempty. We shall call $\Delta=\operatorname{ker} \omega$ a Martinet distribution if it satisfies the following two conditions:

(a) $d H(p) \neq 0$ for all $p \in S$ (then $S$ is smooth), and

(b) $\Delta_{S}=\operatorname{ker} \omega_{S}$ is a quasi-contact structure on $S$, where $\omega_{S}$ is the pullback of $\omega$ to $S$

At each point of $S$ we can define the characteristic line

$$
L_{p}=\left.\operatorname{ker} d \omega_{S}(p)\right|_{\operatorname{ker} \omega_{S}(p)}, \quad p \in S .
$$

The characteristic line field $L=L(\omega)$ for a Martinet distribution $\Delta$ is the field of tangent lines $p \rightarrow L_{p}$ on $S$. It has no singularities. The following fact is a special case of our Theorem 1.4. 
Theorem 0.2. Let $\Delta=\operatorname{ker} \omega$ be a Martinet distribution on a compact orientable manifold $M^{2 k+1}$. If the Martinet hypersurfaces and the characteristic line fields of $\Delta$ and $\tilde{\Delta}=\operatorname{ker} \tilde{\omega}$ are the same and $\tilde{\omega}$ is sufficiently $C^{3}$-close to $\omega$, then there exists a diffeomorphism of the manifold sending $\tilde{\Delta}$ to $\Delta$.

Martinet distributions form a restrictive class of corank one distributions ( $L$ may have singularities). In particular, any Martinet distribution on $M^{3}$ has the Martinet hypersurface $S$, which consists of two-dimensional tori (each connected component of $S$ has zero Euler characteristic, since $S$ admits a 1-dimensional foliation defined by $L$ ). Our Theorem 1.4 will generalize Theorem 0.2 to the case of general corank one distributions and Pfaff equations on $M^{2 k+1}$.

The appearance of the characteristic line field $L$ as one of the main invariants of corank one distributions $\Delta$ has the following history. The first to study $L$ was J. Martinet in Mar for the simplest occurring singularities of $\Delta$ on $\mathbb{R}^{2 n+1}$ (when $L$ has no singularities). J. Martinet also started to study typical singularities of $L$ in the 3-dimensional case. These singularities were roughly classified in [JP], where the existence of a modulus in the classification of characteristic line fields was shown. It was proved in [Zh1] by obtaining a normal form for $\Delta$ that this modulus is the only invariant of $\Delta$. This gave the complete local classification of germs of generic 2-distributions on 3-manifolds, with the characteristic line field as the complete invariant.

In the book [h2] the second author gave a classification of finitely determined singularities of corank one distributions and Pfaff equations on manifolds of any dimension. In this case again the characteristic line field $L$ is a complete invariant. This justifies, to a large extent, making the following

Conjecture. In the space of germs at $0 \in \mathbb{R}^{n}$ of corank one distributions on $\mathbb{R}^{n}$, there is an exceptional set of infinite codimension such that for any two distributions $\Delta_{0}$ and $\Delta_{1}$ away from this set, with the same characteristic line field $L$, there exists a local diffeomorphism $\Phi:\left(\mathbb{R}^{n}, 0\right) \rightarrow\left(\mathbb{R}^{n}, 0\right)$ reducing $\Delta_{1}$ to $\Delta_{0}$.

In a weaker form, with $n=3$, this conjecture has already appeared in JP] and in a letter from J. Martinet to the second author (in 1989). In this case the conjecture can be deduced from the results in JJZ2, JZh1. The exceptional set consists of germs that do not satisfy the assumption (A) or that do not have the property of zeros (see Section 1). In JZh2 we proved that away from the exceptional set the restriction of the distribution to the Martinet hypersurface is a complete invariant for any $n=2 k+1$. If $k=1$, then the restriction can be identified with the characteristic line field.

The results of the present paper concern global corank one distributions close to a fixed distribution. They also deal with families of distributions. In this setting we eliminate some of the difficulties in the above conjecture which are due to non-close germs and the necessity of preserving a fixed point (the source of the germ).

Our transition to the global approach was inspired by the results in Gol] and [MZh, Appendix A]. In [Gol] it is proved that a family of Engel structures $E_{t}$ on a 4-manifold is trivializable provided that the characteristic line field of $E_{t}$ does not depend on $t$. The result in Appendix A of MZh states that two close global corank one distributions of any constant class (in the Cartan-Frobenius sense) are diffeomorphic provided that they have diffeomorphic characteristic foliations. This is a generalization of the Gray theorem. All these results apply to objects without 
singularities, whereas in the present paper we allow any singularities except certain ones of infinite codimension, excluded by the assumption (A). The presence of singularities leads to the main difficulties in our proofs. In Section 2 we explain that the assumption (A) is natural and give examples showing that it cannot be weakened.

In [JZh1] and JZh2] we obtained local realization theorems (for germs at a fixed point), theorems characterizing the set of all possible characteristic line fields if $n=$ 3. Combined with the reduction theorems, they lead to a number of applications including classification results. In this paper we leave aside the difficult task of obtaining global realization theorems. The absence of such theorems restricts, at present, the possibility of drawing immediate interesting conclusions concerning global classification of corank one distributions.

Note that the assumption of closeness which appears in Theorems 0.1 and 0.2 (and later in Theorems 1.2 and 1.4) is essential for our results. Already two contact structures that are far from each other are, in general, not equivalent. A classification of contact structures is known only on certain 3-dimensional manifolds, see e.g. [TEG]. We hope that our results can be used in global contact or quasi-contact geometry for studying singularities of 1-forms which appear when two global contact (or quasi-contact) structures are joined by a path.

There are natural consequences of our results concerning characteristic curves of a distribution, also called singular curves or abnormal curves in sub-Riemannian geometry, and the geometry of distributions (cf. [A], $[\mathrm{BH}],[\mathrm{LS}],[\mathrm{Mon}]$ ). These curves coincide in our case with the integral curves of the characteristic line field.

Under the assumptions of Theorems 0.1 and 0.2, as well as those of Theorems 1.2 and 1.4 in Section 1, the singular curves of a corank 1 distribution determine the equivalence class of the distribution, among distributions close to a given one in the $C^{\infty}$ topology.

\section{Statement of Results}

We will deal with Pfaff equations, which are more general objects than (cooriented) corank one distributions. Let $M^{n}$ denote a compact, orientable, Hausdorff manifold of dimension $n \geq 3$. By definition, a Pfaff equation is a set of differential 1-forms on $M^{n}$ generated, as a module over the ring of functions, by a single 1-form $\omega$. In other words, a Pfaff equation is a 1-form on $M^{n}$ defined up to multiplication by a nonvanishing function. We denote the Pfaff equation by $P=(\omega)$. If $\omega$ vanishes at no points of $M^{n}$, then $(\omega)$ can be identified with the field of kernels of $\omega$ - a coorientable hyperplane field in $T M^{n}$. In general, a Pfaff equation is a more general object, since we do not exclude the possibility of $\omega$ vanishing at some points of the manifold.

All objects in this paper will belong to a fixed category which is either $C^{\infty}$ or real analytic $C^{\omega}$.

The case $n=2 k$. To any Pfaff equation $P$, and in particular to any cooriented corank one distribution, one can associate the characteristic line field.

Definition 1.1. If $n$ is even, $n=2 k$, then any vector field $X$ defined by the relation

$$
X\rfloor \Omega=\omega \wedge(d \omega)^{k-1},
$$


where $\Omega$ is any volume form, and $\omega$ any generator of $P$, is called a characteristic vector field of $P$. The line field on $M^{n}$ generated by $X$, i.e., the mapping

$$
p \rightarrow L_{p}=\{a X(p), a \in \mathbb{R}\} \subset T_{p} M^{2 k},
$$

is called the characteristic line field. The characteristic line field will be denoted by $L$ or $L(\omega)$ or $L(P)$. The set $\left\{p \in M^{2 k}: X(p)=0\right\}$ is called the set of singular points of $L$ and denoted by $\operatorname{Sing}(L)$.

It is easy to check that any two characteristic vector fields differ by multiplication by a nonvanishing function, and consequently the characteristic line field is invariantly related to the Pfaff equation $P$, i.e., the choice of the generator $\omega$ of $P$ and the volume form $\Omega$ is irrelevant. Note that if $\left(\omega \wedge(d \omega)^{k-1}\right)(p) \neq 0$, then the definitions of $L_{p}$ and $L$ coincide with those given in the introduction.

In the presence of singularities we need an invariant that describes a "degree of degeneration" of singular points of a characteristic vector field $X$ (for $n$ odd it was introduced in [JZh2]). First we define an invariant that is slightly stronger than the set $\operatorname{Sing}(L)$.

Let $p \in \operatorname{Sing}(L)$. The local characteristic ideal $I_{p}$ at a point $p \in M^{2 k}$ of a Pfaff equation $P$ is the ideal $I_{p}(X)$ in the ring of function germs at $p$, generated by the coefficients $a_{1}, \ldots, a_{n}$ of a characteristic vector field $X$ of $P$, in some coordinate system near $p$. It is easy to see that the ideal $I_{p}$ is invariantly related to the germ at $p$ of $P=(\omega)$ (the choices of a characteristic vector field and of a local coordinate system are irrelevant). The germ at $p$ of the set $\operatorname{Sing}(L)$ is the zero set of $I_{p}$.

Definition 1.2. If $n=2 k$ and $p \in \operatorname{Sing}(L)$, then we define $d_{p}(P)=d_{p}(X)$ as

$$
d_{p}(P)=\operatorname{depth} I_{p}(X) .
$$

Recall that the depth of a proper ideal $I \subset R$ of a ring $R$ is the maximal length of a regular sequence of elements in $I$. A sequence $a_{1}, \ldots, a_{r} \in I$ is called regular if $a_{1}$ is not a zero divisor in $R$ and, for any $i=2, \ldots, r$, the element $a_{i}$ is not a zero divisor in the quotient ring $R /\left(a_{1}, \ldots, a_{i-1}\right)$, where $\left(a_{1}, \ldots, a_{i-1}\right)$ denotes the ideal generated by $a_{1}, \ldots, a_{i-1}$. By definition, depth $R=\infty$.

Remark. In the analytic category, $d_{p}(P)$ is equal to the codimension in $\mathbb{C}^{n}$ of the germ at $p$ of the set of complex zeros of the ideal $I_{p}$ (i.e., the zero level set of the ideal generated by the complexification of the generators of $I_{p}$ in some local coordinates). This follows from the fact that the complexification does not change the depth of an ideal of analytic function germs (cf. e.g. [E]) and from the equality of the $\operatorname{depth}(I)$ and the codimension of the analytic set of zeros of $I$ for any ideal $I$ of holomorphic function germs.

We introduce the following crucial condition:

$$
d_{p}(P) \geq 3 \text { for any point } p \in \operatorname{Sing}(L) .
$$

This condition is rather weak, in particular generic, as will be explained in Section 2 .

The following theorems hold in the categories $C^{\infty}$ and $C^{\omega}$, with $M$ a compact orientable manifold.

Theorem 1.1. Let $P_{t}, t \in[0,1]$, be a family of Pfaff equations on $M^{2 k}, k \geq 2$, that satisfies the following conditions.

(a) All $P_{t}$ define the same characteristic line field $L=L\left(P_{t}\right)$. 
(b) All $P_{t}$ satisfy condition (A).

Then there exists a family $\Phi_{t}$ of diffeomorphisms of $M^{2 k}$ sending $P_{t}$ to $P_{0}$.

Theorem 1.2. Let $P_{0}=\left(\omega_{0}\right)$ and $P_{1}=\left(\omega_{1}\right)$ be Pfaff equations on $M^{2 k}, k \geq 2$, that have the same characteristic line field $L=L\left(P_{0}\right)=L\left(P_{1}\right)$. Assume that condition (A) holds for $P_{0}$. Then there exists a diffeomorphism $\Phi$ sending $P_{1}$ to $P_{0}$ provided that $\omega_{1}$ is sufficiently close to $\omega_{0}$ in the $C^{\infty}$ topology.

In the above theorems as well as in Theorems 1.3 and 1.4 below, all objects are in the same category $C^{\infty}$ or $C^{\omega}$, including regularity with respect to the parameter $t$. The diffeomorphism in Theorem 1.2 can be taken $C^{\infty}$-close to the identity.

Remark (Closeness of $\omega_{1}$ to $\left.\omega_{0}\right)$ ). In many cases one can present a number $r<\infty$ (depending on $P_{0}$ ) such that the closeness in the $C^{\infty}$ topology in Theorem 1.2 can be replaced by closeness in the $C^{r}$ topology. See Theorem 0.1 and Theorem B.2 in Appendix B.

The case $n=2 k+1$. The most basic invariant of a Pfaff equation $P=(\omega)$ on $M^{2 k+1}$ is the set

$$
S=\left\{p \in M^{2 k+1}:\left(\omega \wedge(d \omega)^{k}\right)(p)=0\right\},
$$

called the Martinet hypersurface, which consists of points at which $\omega$ is not a contact form. This set, invariantly related to $P$, is the zero level of the function

$$
H=\omega \wedge(d \omega)^{k} / \Omega
$$

where $\Omega$ is a volume form.

The ideal $(H)$ of the ring of functions on $M^{2 k+1}$, generated by $H$, is also invariantly related to $P$. It is called the Martinet ideal.

The characteristic line field of $P=(\omega)$ on $M^{2 k+1}$ is defined on the set $S$.

Definition 1.3. Any vector field $X$ on $M^{2 k+1}$ satisfying the relation

$$
X\rfloor \Omega=\omega \wedge(d \omega)^{k-1} \wedge d H \quad \bmod (H),
$$

where $\omega$ is any generator of $P$, and $H$ is any generator of the Martinet ideal, will be called a characteristic vector field of $P$. The line field on $S=\{H=0\}$ defined by the relation

$$
p \rightarrow L_{p}=\{a X(p), a \in \mathbb{R}\}, \quad p \in S,
$$

is called the characteristic line field of $P$. It will be denoted by $L$ or $L(\omega)$ or $L(P)$. The set of singular points of $L$ is defined as $\operatorname{Sing}(L)=\left\{p \in M^{2 k+1}: H(p)=\right.$ $0, X(p)=0\}$.

Above and in the rest of the paper the equality of two objects (vector fields, differential forms) mod $(H)$ means that their difference is divisible over $H$ in the space of objects of the same category. To check that the line field defined above is tangent to $S$, note that the definition of $X$ implies that $X\rfloor d H=0$ at any point of $S=\{H=0\}$ and that $X$ vanishes at any point of $S$ at which the 1-form $d H$ vanishes. Thus $X(p) \in T_{p} S$ at any point $p \in S$ at which $S$ is a smooth hypersurface, and $X$ vanishes at all other points. It is easy to check that the characteristic line field is invariantly related to $P$, i.e., the choices of the generator $\omega$ of $P$, the generator $H$ of the Martinet ideal, and the volume form $\Omega$ are irrelevant.

Note that in the case of the Martinet singularity $\left(p \in S\right.$ and $\left(\omega \wedge(d \omega)^{k-1} \wedge\right.$ $d H)(p) \neq 0)$ the definitions of $L_{p}$ and $L$ coincide with those given in the introduction. 
In order to deal with deeper singularities of $P$, namely those allowing singular points of $L$, we introduce our invariant $d_{p}(P)$ in the case of odd $n$ as follows.

Let $p \in \operatorname{Sing}(L)$. The local characteristic ideal $I_{p}$ at a point $p \in M^{2 k+1}$ of a Pfaff equation $P$ is the ideal $I_{p}(H, X)$ in the ring of function germs at $p$ generated by the germ $H_{p}$ of a generator of the Martinet ideal and the coefficients $a_{1}, \ldots, a_{n}$ of a characteristic vector field $X$ of $P$, in some coordinate system near $p$. The ideal $I_{p}$ is invariantly related to the germ at $p$ of $P=(\omega)$ (the choices of a characteristic vector field, a generator of the Martinet ideal, and of a local coordinate system are irrelevant). The germ at $p$ of the set $\operatorname{Sing}(L)$ is the zero set of $I_{p}$.

Definition 1.4. If $n=2 k+1$ and $p \in \operatorname{Sing}(L)$, then we define

$$
d_{p}(P)=d_{p}(H, X)
$$

as the maximal length of a regular sequence in the characteristic ideal $I_{p}(H, X)$, starting with the germ $H_{p}$ as the first element.

Remarks. (a) In Noetherian rings all maximal regular sequences in $I$ are of the same finite length. Moreover, any regular sequence can be completed to a maximal regular sequence. This implies that in the analytic category, independently of the parity of $n$, we have

$$
d_{p}(P)=\operatorname{depth}\left(I_{p}\right) .
$$

(b) Similarly to the case $n=2 k$, in the analytic category, $d_{p}(P)$ is equal to the codimension in $\mathbb{C}^{n}$ of the germ at $p$ of the set of complex zeros of the complexification of the ideal $I_{p}$.

To formulate our reduction theorem for the most general case, we need two properties of the Martinet ideal $(H)$ : the property of zeros and the extension property.

Definition 1.5. The Martinet ideal $(H)$ has the property of zeros if for any $p \in$ $S=\{H=0\}$ the ideal in the ring of all function germs at $p$ generated by the germ $H_{p}$ of $H$ at $p$ coincides with the ideal in the same ring consisting of function germs vanishing on the germ at $p$ of the set $S=\{H=0\}$.

The property of zeros allows us to identify the Martinet hypersurface $S=\{H=$ $0\}$ with the Martinet ideal. In the case of germs this follows from the definition. Examples where the property of zeros is violated at a point $p$ include:

(a) $H_{p}=H_{1}^{2} H_{2}$, where $H_{1}, H_{2}$ are function germs and $H_{1}(p)=0$;

(b) $H_{p}$ is equivalent to $r^{2}=x_{1}^{2}+\cdots+x_{n}^{2}$;

(c) $H_{p}$ is a flat germ (i.e., the Taylor series of $H$ at $p$ is zero);

(d) $H_{p}$ is a zero divisor in the ring of all germs at $p$.

In case (c) the property of zeros is violated, since $\tilde{H}_{p}=r^{-2} H_{p}$ is smooth and has the same germ of zeros as $H_{p}$ but $\tilde{H}_{p} \notin\left(H_{p}\right)$. Note that (d) is a particular case of (c).

The local version of the property of zeros (Definition 1.5) implies the global version: if a function $f$ on $M$ vanishes on the set $S=\{H=0\}$, then $f$ belongs to the ideal $(H)$. This follows from the fact that division by $H$ or by the germ $H_{p}$ is unique (by (d) the germ $H_{p}$ is not a zero divisor).]

\footnotetext{
${ }^{1}$ In the $C^{\infty}$-category the global and local versions of the property of zeros are equivalent (this follows from the partition of unity). In the real analytic category they are equivalent provided that the sheaf of functions vanishing on $S=\{H=0\}$ is coherent. In the proof "global implies
} 
We also need the extension property of the Martinet hypersurface $S=\{H=0\}$. Denote by $C^{\infty}(M)$ the Fréchet space of smooth functions on $M$, equipped with the topology of convergence together with all derivatives. Let $C^{\infty}(M, S)$ denote its closed subspace of functions that vanish on $S$. We define the space of smooth functions on $S$ as the quotient Fréchet space $C^{\infty}(S)=C^{\infty}(M) / C^{\infty}(M, S)$.

Definition 1.6. We say that $S$ has the extension property if there exists a continuous linear operator $\lambda: C^{\infty}(S) \rightarrow C^{\infty}(M)$ such that $\left.\lambda(f)\right|_{S}=f$ for all $f \in C^{\infty}(S)$.

The extension property automatically holds in the $C^{\omega}$ category, since it holds for any analytic subset $S$ of $M$ (see [BS] for a more general extension theorem). It also holds if we assume that $(H)$ has, locally around any point $p \in S$, a generator that is analytic in some coordinate system.

The following theorems hold in the categories $C^{\infty}$ and $C^{\omega}$, with $M$ compact and orientable.

Theorem 1.3. Let $P_{t}, t \in[0,1]$, be a family of Pfaff equations on $M^{2 k+1}, k \geq 1$, that satisfies the following conditions.

(a) All $P_{t}$ have the same Martinet hypersurface $S$, which has the extension property, and their Martinet ideals have the property of zeros (and consequently are the same).

(b) All $P_{t}$ define the same characteristic line field $L=L\left(P_{t}\right)$.

(c) All $P_{t}$ satisfy condition (A).

Then there exists a family $\Phi_{t}$ of diffeomorphisms of $M^{2 k+1}$ sending $P_{t}$ to $P_{0}$.

Remark. Recall that the extension property of $S$ holds automatically in the $C^{\omega}$ category. We conjecture that in the $C^{\infty}$ category the extension property in Theorem 1.3 also can be omitted. Our proofs show that this is so if the family $P_{t}$ has a generator $\omega_{t}$ that is polynomial in $t$.

Theorem 1.4. Let $P_{0}=\left(\omega_{0}\right)$ and $P_{1}=\left(\omega_{1}\right)$ be Pfaff equations on $M^{2 k+1}, k \geq 1$, which have the same Martinet hypersurface $S=S\left(P_{0}\right)=S\left(P_{1}\right)$ and the same characteristic line field $L=L\left(P_{0}\right)=L\left(P_{1}\right)$. Assume that the Martinet ideal of $P_{0}$ has the property of zeros and $P_{0}$ satisfies condition $(A)$. Then there exists a diffeomorphism $\Phi$ sending $P_{1}$ to $P_{0}$, provided that $\omega_{1}$ is sufficiently close to $\omega_{0}$ in the $C^{\infty}$ topology.

Remark (Closeness of $\omega_{1}$ to $\omega_{0}$ ). As in the even-dimensional case, often one can present a number $r<\infty$ (depending on $P_{0}$ ) such that the closeness in the $C^{\infty}$ topology in Theorem 1.4 can be replaced by closeness in the $C^{r}$ topology. See Theorem 0.2 and Theorem B.2 in Appendix B.

The contents of the further sections. In Section 2 we explain why the condition (A) is natural and give examples showing that it cannot be weakened. The consequences of condition (A) are explained in Section 3 and Appendix A: the condition (A) implies certain global division properties of a characteristic vector field. Section 4 contains auxiliary algebraic statements, which also will be used throughout the proofs. Using the division properties and these algebraic statements, we prove Theorems 1.1 and 1.3 in Sections 5 and 7, respectively. The proofs of these theorems are based on the homotopy method, according to which it suffices to prove

local" one should use Cartan's Theorem A in [C], which says that any local section of a coherent analytic module belongs to the module generated by global sections. 
the solvability of the equation

$$
L_{Z_{t}} \omega_{t}+h_{t} \omega_{t}+\frac{d \omega_{t}}{d t}=0
$$

with respect to a family of vector fields $Z_{t}$ and a family of functions $h_{t}$ (here $L_{Z} \omega$ denotes the Lie derivative of $\omega$ along $Z$ ). Then the family $\Phi_{t}$ of diffeomorphisms obtained by integrating the family of vector fields $Z_{t}$,

$$
\frac{d \Phi_{t}}{d t}=Z_{t}\left(\Phi_{t}\right), \quad \Phi_{0}=i d
$$

transforms the Pfaff equations $\left(\omega_{t}\right)$ into $\left(\omega_{0}\right)$ :

$$
\Phi_{t}^{*} \omega_{t}=\psi_{t} \omega_{0}
$$

where $\psi_{t}=\exp \left(-\int_{0}^{t} \tilde{h}_{s} d s\right)$ and $\tilde{h}_{t}=h_{t} \circ \Phi_{t}$. In what follows the equation (HE) will be called the homotopy equation or homological equation.

Theorems 1.2 and 1.4 are proved in Sections 6 and 8 by reduction to Theorems 1.1 and 1.3. In these sections we show that if Pfaff equations $P_{0}$ and $P_{1}$ satisfy the assumptions of Theorem 1.2 or 1.4, then there exist generators $\omega_{0}$ of $P_{0}$ and $\omega_{1}$ of $P_{1}$ such that the path of Pfaff equations $P_{t}$ generated by $\omega_{t}=\omega_{0}+t\left(\omega_{1}-\omega_{0}\right)$ satisfies the assumptions of Theorem 1.1 or Theorem 1.3.

In Appendix B we present certain topological properties of linear operators related to the Martinet ideal and the characteristic ideal. We also show a way of transition from the assumption of $C^{\infty}$-closeness of $\omega_{1}$ to $\omega_{0}$ in Theorems 1.2 and 1.4 to the $C^{r}$-closeness with a certain $r<\infty$. In the simplest cases this way leads to Theorems 0.1 and 0.2 in the Introduction.

\section{NeCESSity of CONDItion (A)}

In this section we explain why the condition

$$
d_{p}(P) \geq 3
$$

is natural, and we give examples showing that this condition cannot be weakened: if depth $d_{p}(P)=2$, then our theorems are not true anymore.

Fix a point $p \in M^{n}$ and denote by $J_{p}^{i}$ the space of $i$-jets of 1 -forms at $p$. The condition that $p$ is a singular point of the characteristic line field $L$, i.e. $p \in \operatorname{Sing}(L)$, is the condition

$$
\left(\omega \wedge(d \omega)^{k-1}\right)(p)=0
$$

if $n=2 k$, and

$$
\left(\omega \wedge(d \omega)^{k}\right)(p)=0, \quad\left(\omega \wedge(d \omega)^{k-1} \wedge d H\right)(p)=0,
$$

if $n=2 k+1$. It involves the $i$-jet at $p$ of a generator $\omega$ of $P$, where $i=1$ if $n$ is even and $i=2$ if $n$ is odd. This condition distinguishes a certain subset of $J_{p}^{i}$ - the space of $i$-jets of $\omega$ at $p$. It is not difficult to see that for any parity of $n$ this subset is a stratified submanifold of codimension 3 (see $\mathrm{Mar}$, Zh2, or JZh2 for more details). Consequently, for generic $\omega$ the set $\operatorname{Sing}(L)$ is either empty or a submanifold of $M^{n}$ of codimension 3 . In the real analytic category (and conjecturally, in the smooth category too) the set of 1-forms $\omega$ violating (A) has infinite codimension in the space of all 1-forms on $M^{n}$; see JZh2], Proposition 3.4 and Theorem A2 (Appendix 2).

The following examples show that the condition (A) cannot be replaced by the condition $d_{p}(P) \geq 2$. In these examples $\operatorname{dim} M=4$ and $\operatorname{dim} M=5$. They can be 
extended to higher dimensions. We have not found an example in the 3-dimensional case, but we believe that such an example exists.

Example 1. Consider the family of Pfaff equations on the 4-torus $T^{4}$ generated by 1 -forms

$$
\omega_{t}=d \theta_{1}+\left(\sin \theta_{3} \sin \theta_{4}+t\right) d \theta_{2} .
$$

The characteristic vector field $X_{t}$ is the same for all $t$ :

$$
X_{t}=X_{0}=\cos \theta_{4} \sin \theta_{3} \frac{\partial}{\partial \theta_{3}}-\cos \theta_{3} \sin \theta_{4} \frac{\partial}{\partial \theta_{4}} .
$$

The set $S$ of singular points of $X_{0}$ is the union of 8 disjoint 2-dimensional tori (4 of them are described by the equations $\theta_{3}, \theta_{4} \in\{\pi / 2,-\pi / 2\}$, and the other 4 by the equations $\left.\theta_{3}, \theta_{4} \in\{0,-\pi\}\right)$. The codimension of $S$ is 2 ; therefore depth $I_{p}=2$. The restriction of $\left(\omega_{t}\right)$ to any of these 2-dimensional tori is a Pfaff equation generated by a 1 -form $\alpha_{t}=d \theta_{1}+(\delta+t) d \theta_{2}$, where $\delta \in\{0, \pm 1\}$ depending on the torus. This Pfaff equation can be identified with the vector field $V_{t}:(\delta+t) \partial / \partial \theta_{1}-\partial / \partial \theta_{2}$ defined up to multiplication by a nonvanishing function. It follows that the phase portrait of $V_{t}$ on the torus is invariantly related to $\left(\omega_{t}\right)$. It is well known that the equivalence of the phase portraits of $V_{t_{1}}$ and $V_{t_{2}}$ with a fixed $\delta$ implies $t_{1}=t_{2}$ provided that $t_{2}$ is close to $t_{1}$; see ArI] (the parameter $t$ corresponds to the rotation number). Therefore the parameter $t$ of the family $P_{t}$ is a modulus (a parameter varying continuously and distinguishing nonequivalent Pfaff equations).

Example 2. Consider the family of Pfaff equations on the 5-torus

$$
T^{5}\left(\theta_{1}, \theta_{2}, \phi_{1}, \phi_{2}, \phi_{3}\right)
$$

generated by 1 -forms

$$
\omega_{t}=\left(A\left(\theta_{1}, \theta_{2}\right)+B_{t}\left(\theta_{1}, \theta_{2}, \phi_{2}\right)\right) d \phi_{1}+C\left(\theta_{1}, \theta_{2}\right) d \phi_{2}+d \phi_{3},
$$

where

$$
\begin{aligned}
A\left(\theta_{1}, \theta_{2}\right) & =3\left(\sin \theta_{1}+\sin \theta_{2}\right), \\
B_{t}\left(\theta_{1}, \theta_{2}\right) & =t \sin \phi_{2}\left(1-\cos \left(\theta_{1}-\theta_{2}\right)\right), \\
C\left(\theta_{1}, \theta_{2}\right) & =\cos \theta_{1}+\cos \theta_{2} .
\end{aligned}
$$

A simple calculation gives that $\omega_{t} \wedge\left(d \omega_{t}\right)^{2}=\sin \left(\theta_{1}-\theta_{2}\right) \cdot Q_{t} \cdot \Omega$, where $\Omega$ is a volume form and $Q_{t}$ is a family of nonvanishing functions on $T^{5}$, if $t \in[-1,1]$. Therefore the Martinet ideal is the same for all $t$; it is generated by the function $\sin \left(\theta_{1}-\theta_{2}\right)$. The Martinet hypersurface consists of two disjoint 4-tori:

$$
S=T_{1}^{4} \cup T_{2}^{4}, \quad T_{1}^{4}=\left\{\theta_{2}=\theta_{1}\right\}, \quad T_{2}^{4}=\left\{\theta_{2}=\theta_{1}+\pi\right\} .
$$

Since the function $B_{t}$ vanishes on the torus $T_{1}^{4}$, the restriction of $\left(\omega_{t}\right)$ to $T_{1}^{4}$ does not depend on $t$. The restriction of $\left(\omega_{t}\right)$ to the torus $T_{2}^{4}\left(\phi_{1}, \phi_{2}, \phi_{3}, \theta_{1}\right)$ depends on $t$; it is the Pfaff equation $\left(\alpha_{t}\right)$, where $\left(\alpha_{t}\right)=2 t \sin \phi_{2} d \phi_{1}+d \phi_{3}$. The characteristic vector field of $\left(\omega_{t}\right)$ restricted to $T_{2}^{4}$ is the characteristic vector field of $\left(\alpha_{t}\right)$. It is $2 t \cos \phi_{2} \partial / \partial \theta_{1}$. Assume that $t \neq 0$. Then the characteristic line field does not depend on $t$. The set of its singular points is the union of two disjoint 3 -tori $T_{ \pm}^{3}$, given by the equations $\phi_{2}= \pm \pi / 2$. The restriction of $\left(\omega_{t}\right)$ to $T_{ \pm}^{3}$ (or, the same, the restriction of $\left(\alpha_{t}\right)$ to $\left.T_{ \pm}^{3}\right)$ is the Pfaff equation of the form $\left(\beta_{t}\right), \beta_{t}=2 t \delta d \phi_{1}+d \phi_{3}$, $\delta \in\{-1,1\}$. Consider the vector field $V_{t}:-\partial / \partial \phi_{1}+2 t \delta \partial / \partial \phi_{3}$ on the 2 -torus $T^{2}=T^{2}\left(\phi_{1}, \phi_{3}\right)$. It is easy to see that the Pfaff equations $\left(\beta_{t_{1}}\right)$ and $\left(\beta_{t_{2}}\right)$ on the 
3 -torus are equivalent if and only if the phase portraits of $V_{t_{1}}$ and $V_{t_{2}}$ on the 2torus are equivalent. As in the previous example, this is so if and only if $t_{1}=t_{2}$ provided that $t_{2}$ is close to $t_{1}$. Therefore the parameter $t$ of the family $\left(\omega_{t}\right)$ of Pfaff equations is a modulus, although these Pfaff equations have the same Martinet ideal (satisfying the property of zeros) and the same characteristic line field. The reason for that is the violation of the assumption $(\mathrm{A})$ - the depth of the characteristic ideal is equal to 2 instead of 3 .

\section{Condition (A) And Division properties}

In this section we explain implications of condition (A) which will be essential in further proofs. The main implications are the following global division properties of a characteristic vector field $X$. As before, we work in the $C^{\infty}$ and $C^{\omega}$ categories.

Proposition 3.1.a. If a Pfaff equation $P=(\omega)$ satisfies condition $(A)$, then any characteristic vector field $X$ of $P$ has the following division properties.

(i) If $n$ is even, then for any vector field $Y$ and any $r$-form $\nu$ on $M^{n}$ with $r=n-1$ or $r=n-2$ the equality

$$
X\rfloor \nu=0 \quad \text { implies } \quad \nu=X\rfloor \mu,
$$

for an $(r+1)$-form $\mu$ on $M^{n}$, and the equality

$$
X \wedge Y=0 \quad \text { implies } \quad Y=f X
$$

for a function $f$ on $M^{n}$.

(ii) If $n$ is odd, then for any vector field $Y$ on $M^{n}$ and any $(n-1)$-form $\nu$ on $M^{n}$ the equality

$$
X\rfloor \nu=0 \quad \bmod (H) \quad \text { implies } \quad \nu=X\rfloor \mu \bmod (H),
$$

for an $n$-form $\mu$ on $M^{n}$, and the equality

$$
X \wedge Y=0 \bmod (H) \quad \text { implies } Y=f X \bmod (H)
$$

for a function $f$ on $M^{n}$. Here $(H)$ is the Martinet ideal of $P$, and we assume that $(H)$ has the property of zeros.

This proposition is a corollary of a general theorem in [DJ] on division properties of the interior product with a section $X$ of a vector bundle (see Appendix A for the proof).

We also need a division property with parameters. In the next and all further statements in this section a 1-parameter family of functions, differential forms or vector fields on $M$ is assumed to be regular in $t$, i.e., depending on $t$ analytically (in the $C^{\omega}$ category) or smoothly (in the $C^{\infty}$ category).

Proposition 3.1.b. Proposition 3.1.a holds with the forms $\nu, \mu$, vector field $Y$, and function $f$ replaced by families $\nu_{t}, \mu_{t}, Y_{t}, f_{t}, t \in[0,1]$, provided that in the odd-dimensional case either the set $S=\{H=0\}$ has the extension property (see Section 1) or the families $\nu_{t}$ and $Y_{t}$ depend on $t$ polynomially.

This proposition is also proved in Appendix A, using the already mentioned general theorem on division properties.

Remark. Proposition 3.1 also holds for germs at a fixed point.

Another implication of condition (A) concerns the structure of the set $\operatorname{Sing}(L)$ of singular points of the characteristic foliation $L$ : it cannot be too degenerate. 
Proposition 3.2. If a Pfaff equation ( $\omega)$ satisfies condition (A) and in the odddimensional case the Martinet ideal of $(\omega)$ has the property of zeros, then any characteristic vector field $X$ of $(\omega)$, the Martinet hypersurface $S$ and the set Sing $(L)$ of singular points of the characteristic foliation have the following properties.

(i) If $n=2 k$, then the set $M^{n} \backslash \operatorname{Sing}(L)$ of points where $X$ does not vanish (i.e. ( $\omega)$ is quasi-contact) is dense in $M^{n}$.

(ii) If $n=2 k+1$, then the set $M^{n} \backslash S$ (i.e., the set of points at which $\omega$ is contact) is dense in $M^{n}$. Equivalently, any generator $H$ of the Martinet ideal is not a zero divisor.

(iii) If $n=2 k+1$, then the set $S \backslash \operatorname{Sing}(L)$ is dense in $S$.

Proof. Statement (i) follows from the observation that if $\omega$ is not quasi-contact at any point of an open set, then any characteristic vector field $X$ vanishes on this set (vanishes on a connected component of $M^{n}$, in the analytic category). Consequently, given a point $p$ in this set, the characteristic ideal $I_{p}$ at $p$ generated by the coefficients of $X$ is trivial and contains no non-zero-divisor. This contradicts assumption (A).

Statement (ii) is a simple implication of the property of zeros of the Martinet ideal; see Definition 1.5 and the examples following it.

To prove (iii), assume that there exists a neighbourhood $U$ in $M^{n}$ of a point $p \in S$ such that a characteristic vector field $X$ vanishes at any point of the set $U \cap S$. By the property of zeros of the Martinet ideal we obtain that $X_{p}=0 \bmod \left(H_{p}\right)$, where the subscript indicates the germ at $p$. This contradicts assumption (A) at the point $p$. The proof is complete.

Propositions 3.1 and 3.2 imply the possibility of choosing the same characteristic vector field for all Pfaff equations with the same characteristic foliation.

Proposition 3.3. Let $P_{t}=\left(\omega_{t}\right), t \in[0,1]$, be a family of Pfaff equations on $M^{n}$ satisfying the assumptions of Theorem 1.1, if $n=2 k$, or of Theorem 1.3, if $n=2 k+1$. Then for any family $X_{t}$ of characteristic vector fields of $\left(\omega_{t}\right)$ we have

$$
\begin{aligned}
& X_{t}=R_{t} X_{0}, \quad \text { if } n=2 k \text {, or } \\
& X_{t}=R_{t} X_{0} \quad \bmod (H), \quad \text { if } \quad n=2 k+1 \text {, }
\end{aligned}
$$

where $R_{t}, t \in[0,1]$, is a family of positive-valued functions and $X_{0}$ is $X_{t}$ with $t=0$.

Proof. Let $n=2 k$. The equality $L\left(\omega_{t}\right)=L\left(\omega_{0}\right)$ implies that $\left(X_{t} \wedge X_{0}\right)(p)=0$ for all $p \in M^{n}$. By Proposition 3.1.b we obtain that $X_{t}=R_{t} X_{0}$, where $R_{t}$ is a family of functions. Proposition 3.1.b also implies that for any fixed $t$ we have $X_{0}=Q_{t} X_{t}$, where $Q_{t}$ is a function on $M^{n}$. This leads to the relation $\left(1-R_{t} Q_{t}\right) X_{0}=0$. By Proposition 3.2, $X_{0}$ does not vanish on a dense subset of $M^{n}$; thus $R_{t} Q_{t}=1$. This implies that $R_{t}$ is a family of nonvanishing functions. This family is positive valued, since for any $p \in M^{n}$ the function $R_{t}(p)$ is continuous in $t$ and $R_{0}(p)=1$.

In the case of $n=2 k+1$ the equality $L\left(\omega_{t}\right)=L\left(\omega_{0}\right)$ gives $\left(X_{t} \wedge X_{0}\right)(p)=0$ for all $p \in S$. From the property of zeros of the Martinet ideal we deduce that $X_{t} \wedge X_{0}=0 \bmod (H)$. Using Proposition 3.1.b, we see that $X_{t}=R_{t} X_{0} \bmod (H)$, for a family of functions $R_{t}$. Similarly, we have $X_{0}=Q_{t} X_{t} \bmod (H)$ for any fixed $t$, where the $Q_{t}$ are functions. Therefore, $\left(1-R_{t} Q_{t}\right) X_{0}=0 \bmod (H)$. By Proposition $3.2, R_{t} Q_{t}=1$ on $S$, and so $R_{t}$ is nonvanishing on $S$. From the fact that $R_{t}(p)$ is continuous in $t$ and from $R_{0}(p)=1$, we deduce that $R_{t}$ is positive valued on $S$, 
for any $t \in[0,1]$. Finally, adding to $R_{t}$ the function $C H^{2}$ with a sufficiently large constant $C$, we obtain $R_{t}$ positive valued on $M$.

Condition (A) implies one more division property that we need in our proofs. Its proof is postponed to Section 4.

Proposition 3.4. Let $P_{t}=\left(\omega_{t}\right), t \in[0,1]$, be a family of Pfaff equations on $M^{2 k}$ satisfying the assumptions of Theorem 1.1, and let $\beta_{t}$ be a family of 1-forms such that $\omega_{t} \wedge \beta_{t}=0$. Then $\beta_{t}=h_{t} \omega_{t}$ for some family $h_{t}$ of functions.

Note that this statement is trivial if $\omega_{t}$ is a family of nonvanishing 1-forms, but we do not assume this in our theorems.

\section{Auxiliary algebraic lemmas}

To prove the solvability of the homotopy equation in the proofs of Theorems 1.1 and 1.3, we will also use the following simple algebraic facts.

Recall that a 1 -form $\alpha$ on $M^{n}, n \geq 3$, is called contact (quasi-contact) at $p \in$ $M^{n}$ if $n=2 k+1$ (respectively, $\left.n=2 k\right)$ and $\left(\alpha \wedge(d \alpha)^{k}\right)(p) \neq 0$ (respectively, $\left.\left(\alpha \wedge(d \alpha)^{k-1}\right)(p) \neq 0\right)$.

Lemma 4.1. Let $\alpha$ and $\lambda$ be 1-forms on $M^{2 k}$. If $\alpha$ is quasi-contact at $p$ and $\left(\lambda \wedge \alpha \wedge(d \alpha)^{k-2}\right)(p)=0$, then $(\lambda \wedge \alpha)(p)=0$.

Lemma 4.2. Let $\alpha$ be $a 1$-form on $M^{2 k+1}$. If $\alpha$ is a contact at $p$ and $\lambda$ is a 1-form such that $\left(\lambda \wedge \alpha \wedge(d \alpha)^{k-1}\right)(p)=0$ and $\left(\lambda \wedge(d \alpha)^{k}\right)(p)=0$, then $\lambda(p)=0$.

The facts stated in these lemmas are invariant with respect to multiplication of $\alpha$ by a nonvanishing function, i.e., they are properties of the Pfaff equation $(\alpha)$. These properties can be easily checked in the Darboux coordinates in which the Pfaff equation takes the form $\left(d z+x_{1} d y_{1}+\cdots+x_{r} d y_{r}\right)$, where $r=k-1$ if $n=2 k$, and $r=k$ if $n=2 k+1$.

Lemma 4.3. Let $\alpha$ be a 1 -form on $M^{2 k+1}$ that is not contact at $p$, but $\alpha(p) \neq 0$. If $\lambda$ is a 1-form such that $\left(\lambda \wedge \alpha \wedge(d \alpha)^{k-1}\right)(p)=0$, then $\left(\lambda \wedge(d \alpha)^{k}\right)(p)=0$.

Proof. We take a nonzero vector $v \in T_{p} M^{2 k+1}$ such that $\left.\left.v\right\rfloor \alpha=v\right\rfloor d \alpha=0$. (The existence of such a vector follows from the assumption that $\alpha$ is not contact at $p$.) Then the relation assumed in the lemma implies that the form $(v\rfloor \lambda) \cdot \alpha \wedge(d \alpha)^{k-1}$ vanishes at $p$. It follows that if $\left(\alpha \wedge(d \alpha)^{k-1}\right)(p) \neq 0$, then $(v \mid \lambda)(p)=0$, and consequently $\left(\lambda \wedge(d \alpha)^{k}\right)(p)=0$. On the other hand, if $\left(\alpha \wedge(d \alpha)^{k-1}\right)(p)=0$, then the assumption $\alpha(p) \neq 0$ implies that $(d \alpha)^{k}(p)=0$, and then again $\left(\lambda \wedge(d \alpha)^{k}\right)(p)=$ 0 .

Lemma 4.4. If $\Omega$ is a volume form on $M^{n}, \lambda$ is a 1-form, $\gamma$ is an $(n-2)$-form and $X$ is a vector field defined by the relation $X\rfloor \Omega=\lambda \wedge \gamma$, then $X\rfloor \lambda=0$.

Proof. To prove this statement, note that the definition of $X$ implies $X\rfloor(\lambda \wedge \gamma)=0$, and consequently $(X\rfloor \lambda) \cdot \gamma \pm(X\rfloor \gamma) \wedge \lambda=0$. It follows that $(X\rfloor \lambda) \cdot(\lambda \wedge \gamma)=0$. Since $X$ vanishes exactly at points at which the form $\lambda \wedge \gamma$ vanishes, we obtain that $X\rfloor \lambda=0$.

Finally, we need the following general properties of a characteristic vector field. 
Lemma 4.5. If $X$ is a characteristic vector field of a Pfaff equation ( $\omega)$ on $M^{2 k}$, then

$$
X\rfloor \omega=0 \quad \text { and } \quad(X\rfloor d \omega) \wedge \omega=0 .
$$

Proof. The first relation follows from the definition of $X$ and Lemma 4.4. The definition of $X$ implies that $X\rfloor\left(\omega \wedge(d \omega)^{k-1}\right)=0$, which, together with $\left.X\right\rfloor \omega=0$, gives $(X\rfloor d \omega) \wedge \omega \wedge(d \omega)^{k-2}=0$ if $k>1$. Now the second relation in Lemma 4.5 follows from Lemma 4.1 at points where $\omega$ is quasi-contact. At all other points the field $X$ vanishes, and there is nothing to prove.

Lemma 4.6. If $X$ is a characteristic vector field of a Pfaff equation ( $\omega)$ on $M^{2 k+1}$ whose Martinet ideal has the property of zeros, and $H$ is a generator of this ideal, then

$$
X\rfloor \omega=0 \quad \bmod (H), \quad X\rfloor d H=0 \quad \bmod (H), \quad(X\rfloor d \omega) \wedge \omega=0 \quad \bmod (H) .
$$

Proof. Due to the property of zeros of $(H)$, it suffices to prove the three relations at any point $p$ of the Martinet hypersurface $S$ such that $X(p) \neq 0$. The relation $(X\rfloor d H)(p)=0$ follows immediately from the definition of $X$. To see the other two relations, note that $S$ is regular in a neighbourhood of a point $p$ such that $X(p) \neq 0$. The definition of the characteristic vector field $X$ in the case $n=2 k+1$ implies that the vector field $\left.X\right|_{S}$ on $S$ is, in a neighbourhood of such a point $p$, a characteristic vector field of the Pfaff equation $\left(\left.\omega\right|_{S}\right)$ on $S$ (which is quasi-contact at $p$ ). Thus the remaining two relations follow from Lemma 4.5. The proof is complete.

Proof of Proposition 3.4. Let $X_{t}$ be the characteristic vector field of $\left(\omega_{t}\right)$ defined by $\left.X_{t}\right\rfloor \Omega=\omega_{t} \wedge\left(d \omega_{t}\right)^{k-1}$. Since $X_{t}$ (and so $\left.\omega_{t}\right)$ does not vanish on a dense subset of $M^{n}$, the condition $\omega_{t} \wedge \beta_{t}=0$ and Lemma 4.5 imply that $\left.X_{t}\right\rfloor \beta_{t}=0$ and $\left.X_{t}\right\rfloor\left(\beta_{t} \wedge\left(d \omega_{t}\right)^{k-1}\right)=0$. From Proposition 3.3 we have the equality $X_{t}=R_{t} X_{0}$, with $R_{t}$ nonvanishing; thus $\left.X_{0}\right\rfloor\left(\beta_{t} \wedge\left(d \omega_{t}\right)^{k-1}\right)=0$. Therefore the division property in Proposition 3.1.b implies the following relation: $\left.\beta_{t} \wedge\left(d \omega_{t}\right)^{k-1}=X_{0}\right\rfloor \mu_{t}=$ $\left.\left(g_{t} / R_{t}\right) X_{t}\right\rfloor \Omega$, where $\Omega$ is a volume form, $g_{t}$ is a family of functions and $\mu_{t}=g_{t} \Omega$. Taking $h_{t}=g_{t} / R_{t}$, we can rewrite this relation in the form

$$
\left(\beta_{t}-h_{t} \omega_{t}\right) \wedge\left(d \omega_{t}\right)^{k-1}=0 .
$$

Let us show that this relation implies $\beta_{t}-h_{t} \omega_{t}=0$. We know that $\left(\beta_{t}-h_{t} \omega_{t}\right) \wedge \omega_{t}=$ 0 , since $\beta_{t} \wedge \omega_{t}=0$. Fix $t$ and a point $p$ at which $\omega_{t}$ is quasi-contact. At this point $\omega_{t}$ does not vanish; therefore $\left(\beta_{t}-h_{t} \omega_{t}\right)(p)=r \omega_{t}(p)$, with the scalar $r$ depending on $t$ and $p$. Then the displayed relation implies that $r\left(\omega_{t} \wedge\left(d \omega_{t}\right)^{k-1}\right)(p)=0$ and consequently $r=0$. So, $\left(\beta_{t}-h_{t} \omega_{t}\right)(p)=0$ if $p$ is a point at which $\omega_{t}$ is quasicontact. By Proposition 3.2, (i) the set of such points is dense, and so $\beta_{t}=h_{t} \omega_{t}$ at any point of the manifold. The proof is complete.

Now we are ready to prove the solvability of the homotopy equation (HE) and our main theorems.

\section{Proof of Theorem 1.1}

Solvability of the homotopy equation (HE) in Section 1 is equivalent to solvability of

$$
\left(L_{Z_{t}} \omega_{t}+\frac{d \omega_{t}}{d t}\right) \wedge \omega_{t} \wedge\left(d \omega_{t}\right)^{k-2}=0
$$


with respect to a family $Z_{t}$ of vector fields. Namely, equation (5.1) is obtained from the homotopy equation by external multiplication by $\omega_{t} \wedge\left(d \omega_{t}\right)^{k-2}$. Conversely, if (5.1) is solvable then, using the fact that the set of quasi-contact points of $\left(\omega_{t}\right)$ is dense in $M^{n}$ (Proposition 3.2,(i)), we get from (5.1) by Lemma 4.1 that $\left(L_{Z_{t}} \omega_{t}+\right.$ $\left.\left(d \omega_{t} / d t\right)\right) \wedge \omega_{t}=0$. Therefore, by Proposition 3.4 we get $L_{Z_{t}} \omega_{t}+\left(d \omega_{t} / d t\right)+h_{t} \omega_{t}=0$, for a family of functions $h_{t}$, which is the homotopy equation (HE).

A solution $Z_{t}$ of equation (5.1) will be constructed within the set of families $Z_{t}$ satisfying

$$
\left.Z_{t}\right\rfloor \omega_{t}=0 .
$$

Condition (5.2) implies that $\left.L_{Z_{t}} \omega_{t}=Z_{t}\right\rfloor d \omega_{t}$, and the equation (5.1) can be rewritten in the form

$$
\left.Z_{t}\right\rfloor\left(\omega_{t} \wedge\left(d \omega_{t}\right)^{k-1}\right)+(k-1) \frac{d \omega_{t}}{d t} \wedge \omega_{t} \wedge\left(d \omega_{t}\right)^{k-2}=0 .
$$

In order to solve equation (5.3) we fix a volume form $\Omega$ and define a family $X_{t}$ of characteristic vector fields of $\left(\omega_{t}\right)$ by the relation $\left.X_{t}\right\rfloor \Omega=\omega_{t} \wedge\left(d \omega_{t}\right)^{k-1}$. Lemma 4.5 and Proposition 3.3 imply the relations $\left.\left.X_{0}\right\rfloor \omega_{t}=0, X_{0}\right\rfloor\left(d \omega_{t} / d t\right)=0$ and $\left.\left(X_{0}\right\rfloor d \omega_{t}\right) \wedge \omega_{t}=0$. Thus

$$
\left.X_{0}\right\rfloor \nu_{t}=0, \quad \text { where } \quad \nu_{t}=\left(\frac{d \omega_{t}}{d t} \wedge \omega_{t} \wedge\left(d \omega_{t}\right)^{k-2}\right) .
$$

Therefore, by the division property in Proposition 3.1.b, we have

$$
\left.\nu_{t}=X_{0}\right\rfloor \tilde{\mu}_{t},
$$

with some family $\tilde{\mu}_{t}$ of $(n-1)$-forms of the same regularity with respect to $t$ as in $\omega_{t}$. Using Proposition 3.3 again, we obtain

$$
\left.\nu_{t}=X_{t}\right\rfloor \mu_{t}
$$

for some, regular in $t$, family of $(n-1)$-forms $\mu_{t}$. This relation allows to rewrite equation (5.3) in the form

$$
\left.\left.\left.Z_{t}\right\rfloor\left(X_{t}\right\rfloor \Omega\right)+(k-1) X_{t}\right\rfloor \mu_{t}=0 .
$$

The latter equation has a solution $Z_{t}$ defined by the relation

$$
\left.Z_{t}\right\rfloor \Omega=(k-1) \mu_{t} .
$$

It now remains to check that the constructed solution $Z_{t}$ satisfies relation (5.2). The equality (5.2) is equivalent to the relation $\mu_{t} \wedge \omega_{t}=0$. From (5.5) and the definition of $\nu_{t}$ we have $\left.\left(X_{t}\right\rfloor \mu_{t}\right) \wedge \omega_{t}=0$. By Lemma 4.5, $\left.X_{t}\right\rfloor \omega_{t}=0$; therefore $\left.X_{t}\right\rfloor\left(\mu_{t} \wedge \omega_{t}\right)=0$. Thus the $n$-form $\mu_{t} \wedge \omega_{t}$ vanishes at any point at which $X_{t}$ does not vanish. By Proposition 3.2, (i) the set of such points is everywhere dense; therefore $\mu_{t} \wedge \omega_{t}=0$ and (5.2) holds. This completes the proof of Theorem 1.1.

\section{Proof of Theorem 1.2}

We will use the following proposition (its proof is postponed to the end of this section).

Proposition 6.1. Assume that $P_{0}=\left(\omega_{0}\right)$ satisfies condition ( $\left.A\right)$ and

$$
\omega_{1} \wedge\left(d \omega_{1}\right)^{k-1}=\omega_{0} \wedge\left(d \omega_{0}\right)^{k-1} .
$$


Then for the path $\omega_{t}=(1-t) \omega_{0}+t \omega_{1}$ we have

$$
\omega_{t} \wedge\left(d \omega_{t}\right)^{k-1}=A_{t} \omega_{0} \wedge\left(d \omega_{0}\right)^{k-1},
$$

where $A_{t}$ is a family of functions, polynomial in $t$.

Proof of Theorem 1.2. The equality $L\left(\omega_{0}\right)=L\left(\omega_{1}\right)$ implies $X_{0} \wedge X_{1}=0$, where $X_{0}$ and $X_{1}$ are characteristic vector fields of $P_{0}$ and $P_{1}$. Condition (A) satisfied for $\left(\omega_{0}\right)$ enables us to use the second division property in Proposition 3.1.a, (i) to deduce that $X_{1}=R X_{0}$ and, equivalently,

$$
\omega_{1} \wedge\left(d \omega_{1}\right)^{k-1}=R \omega_{0} \wedge\left(d \omega_{0}\right)^{k-1},
$$

where $R$ is a smooth or analytic function. In fact, $R$ is positive valued, which will follow from the closeness of $\omega_{1}$ to $\omega_{0}$. Therefore, assuming $R>0$, we choose the generator

$$
\hat{\omega}_{1}=R^{1 / k} \omega_{1},
$$

and we have

$$
\hat{\omega}_{1} \wedge\left(d \hat{\omega}_{1}\right)^{k-1}=\omega_{0} \wedge\left(d \omega_{0}\right)^{k-1} .
$$

Let

$$
\omega_{t}=(1-t) \omega_{0}+t \hat{\omega}_{1} .
$$

To prove Theorem 1.2 it is sufficient to show that the family of Pfaff equations $\left(\omega_{t}\right)$ satisfies the assumptions of Theorem 1.1.

The equality (6.4) allows us to use Proposition 6.1 to conclude that the relation (6.2) holds for the path (6.5). It is clear that (6.2) implies that the family $\left(\omega_{t}\right)$ satisfies the assumptions (a) and (b) of Theorem 1.1 provided that the functions $A_{t}$ in $(6.2), t \in[0,1]$, vanish at no point of $M^{n}$. This will follow from the assumption on the $C^{\infty}$-closeness of $\omega_{1}$ to $\omega_{0}$ and Theorem B1 in Appendix B. Define a characteristic vector field $X_{t}$ of $\left(\omega_{t}\right)$ by the relation $\left.X_{t}\right\rfloor \Omega=\omega_{t} \wedge\left(d \omega_{t}\right)^{k-1}$, where $\Omega$ is a volume form. By (6.2) we have $X_{t}=A_{t} X_{0}$. The $C^{\infty}$-closeness of $\omega_{1}$ to $\omega_{0}$ implies the $C^{\infty}$-closeness of $X_{t}, t \in[0,1]$, to $X_{0}$. By Theorem B1 the $C^{\infty}$-closeness of $X_{t}$ to $X_{0}$ in the equality $X_{t}=A_{t} X_{0}$ implies that the function $A_{t}, t \in[0,1]$, is $C^{\infty}$-close to 1 . Consequently, $A_{t}$ vanishes at no point of the manifold. The proof of Theorem 1.2 is complete.

Proof of Proposition 6.1. Using (6.1), we may assume that the characteristic vector fields $X_{0}$ and $X_{1}$ of $\left(\omega_{0}\right)$ and $\left(\omega_{1}\right)$, respectively, are equal. We shall prove that

$$
\left.X_{0}\right\rfloor\left(\omega_{t} \wedge\left(d \omega_{t}\right)^{k-1}\right)=0 .
$$

Having (6.6), we can use assumption (A) and the division property in Proposition 3.1.b (with polynomial dependence in $t$ ), which gives $\left.\omega_{t} \wedge\left(d \omega_{t}\right)^{k-1}=X_{0}\right\rfloor \mu_{t}$, where $\mu_{t}$ is a volume form. Let $\left.\omega_{0} \wedge\left(d \omega_{0}\right)^{k}=X_{0}\right\rfloor \Omega, \mu_{t}=A_{t} \Omega$. Then we get (6.2).

To prove (6.6), we note that by Lemma 4.5 we have

$$
\left.\left.X_{0}\right\rfloor \omega_{0}=X_{0}\right\rfloor \omega_{1}=0 ;
$$

therefore $\left.X_{0}\right\rfloor \omega_{t}=0$. It follows that in order to prove (6.6) it suffices to prove the equality

$$
\left.\left(X_{0}\right\rfloor d \omega_{t}\right) \wedge \omega_{t}=0
$$

It is enough to prove the equality $(6.8)$ at any point $p$ such that $X_{0}(p) \neq 0$. At such a point $\omega_{0}(p) \neq 0$ and, since $X_{0}=X_{1}, \omega_{1}(p) \neq 0$. From Lemma 4.5 we have 
$\left.\left(X_{0}\right\rfloor d \omega_{i}\right) \wedge \omega_{i}=0, i=0,1$. Thus, there are functions $h_{0}$ and $h_{1}$, defined in a neighbourhood of $p$, such that in this neighbourhood we have

$$
\left.\left.X_{0}\right\rfloor d \omega_{0}=h_{0} \omega_{0}, \quad X_{0}\right\rfloor d \omega_{1}=h_{1} \omega_{1} .
$$

We will prove below that

$$
h_{1}=h_{0} .
$$

Then from (6.9) we get $\left.X_{0}\right\rfloor d \omega_{t}=h \omega_{t}$, where $h=h_{0}=h_{1}$, and so (6.8) holds.

We will show that (6.10) follows from (6.1). We take the Lie derivative of both parts in (6.1) along the vector field $X_{0}$. Using the formula $\left.\left.L_{X} \eta=d(X\rfloor \eta\right)+X\right\rfloor d \eta$ for the Lie derivative, we obtain

$$
\begin{gathered}
\left.\left.L_{X_{0}}\left(\omega_{0} \wedge\left(d \omega_{0}\right)^{k-1}\right)=d\left(X_{0}\right\rfloor\left(\omega_{0} \wedge\left(d \omega_{0}\right)^{k-1}\right)\right)+X_{0}\right\rfloor\left(d \omega_{0}\right)^{k} \\
\left.=0+k\left(X_{0}\right\rfloor d \omega_{0}\right) \wedge\left(d \omega_{0}\right)^{k-1}=k h_{0} \omega_{0} \wedge\left(d \omega_{0}\right)^{k-1},
\end{gathered}
$$

and similarly

$$
L_{X_{0}}\left(\omega_{1} \wedge\left(d \omega_{1}\right)^{k-1}\right)=k h_{1} \omega_{1} \wedge\left(d \omega_{1}\right)^{k-1} .
$$

Comparing these equalities and using (6.1) again, we get the required relation (6.10) (since $\omega_{0} \wedge\left(d \omega_{0}\right)^{k-1}=\omega_{1} \wedge\left(d \omega_{1}\right)^{k-1}$ does not vanish on a dense subset of $\left.M\right)$. Proposition 6.1 is proved.

\section{Proof of Theorem 1.3}

Since the Martinet hypersurfaces of $P_{t}=\left(\omega_{t}\right)$ are the same for all $t$, the Martinet ideals are the same by the property of zeros. Thus we can fix a generator $H$ of these ideals. The following two propositions will hold under the assumptions of Theorem 1.3. In the propositions all families are regular with respect to $t$ (smooth in the $C^{\infty}$ category and analytic in the $C^{\omega}$ category).

Proposition 7.1. There exists a family of vector fields $Y_{t}$ satisfying the relation

$$
\left(L_{Y_{t}} \omega_{t}+\frac{d \omega_{t}}{d t}\right) \wedge \omega_{t} \wedge\left(d \omega_{t}\right)^{k-1}=0 \quad \bmod (H) .
$$

Proposition 7.2. Let $\mu_{t}$ be a family of 1-forms such that

$$
\mu_{t} \wedge \omega_{t} \wedge\left(d \omega_{t}\right)^{k-1}=0 \quad \bmod (H) .
$$

Then the equation

$$
L_{Z_{t}} \omega_{t}+h_{t} \omega_{t}=\mu_{t}
$$

has a solution $\left(Z_{t}, h_{t}\right)$.

The solvability of the homotopy equation (HE) in Section 1 is a direct corollary of these propositions. Namely, we take $\mu_{t}=-L_{Y_{t}} \omega_{t}-d \omega_{t} / d t$, and then the pair $\left(\tilde{Z}_{t}, h_{t}\right)$, with $\tilde{Z}_{t}=Z_{t}+Y_{t}$, solves the homotopy equation (HE).

Proof of Proposition 7.1. We fix a volume form $\Omega$ and define a family $X_{t}$ of characteristic vector fields of $\left(\omega_{t}\right)$ by the relation $\left.X_{t}\right\rfloor \Omega=\omega_{t} \wedge\left(d \omega_{t}\right)^{k-1} \wedge d H$. From Proposition 3.3 we have

$$
X_{t}=R_{t} X_{0} \quad \bmod (H),
$$

where $R_{t}$ is a family of nonvanishing functions, regular in $t$ (of the same regularity in $t$ as in the family $\left.\omega_{t}\right)$. By Lemma 4.6 we have $\left.X_{t}\right\rfloor \omega_{t}=0 \bmod (H)$ and $\left.\left(X_{t}\right\rfloor d \omega_{t}\right) \wedge \omega_{t}=0 \bmod (H)$. We may replace $X_{t}$ with $X_{0}$ in these equalities. In 
particular, we get $\left.X_{0}\right\rfloor \omega_{t}=0 \bmod (H)$, which implies $\left.X_{0}\right\rfloor\left(d \omega_{t} / d t\right)=0 \bmod (H)$. Taking all these equalities into account, we see that

$$
\left.X_{0}\right\rfloor\left(\frac{d \omega_{t}}{d t} \wedge \omega_{t} \wedge\left(d \omega_{t}\right)^{k-1}\right)=0 \quad \bmod (H) .
$$

This equality and Proposition 3.1.b imply that

$$
\left.\frac{d \omega_{t}}{d t} \wedge \omega_{t} \wedge\left(d \omega_{t}\right)^{k-1}=X_{0}\right\rfloor\left(f_{t} \Omega\right) \bmod (H),
$$

where $f_{t}$ is a family of functions, regular in $t$. Replacing $X_{0}$ with $R_{t}^{-1} X_{t}$ and using the definition of $X_{t}$, we see that we can rewrite this relation in the form

$$
\frac{d \omega_{t}}{d t} \wedge \omega_{t} \wedge\left(d \omega_{t}\right)^{k-1}=g_{t} \omega_{t} \wedge\left(d \omega_{t}\right)^{k-1} \wedge d H \quad \bmod (H),
$$

where $g_{t}=f_{t} / R_{t}$. This allows us to rewrite equation (7.1) in the form

$$
\left(L_{Y_{t}} \omega_{t}-g_{t} d H\right) \wedge \omega_{t} \wedge\left(d \omega_{t}\right)^{k-1}=0 \quad \bmod (H) .
$$

It is clear that (7.5) holds if $Y_{t}$ satisfies the relations

$$
\left.\left.Y_{t}\right\rfloor d \omega_{t}=0, \quad Y_{t}\right\rfloor \omega_{t}=g_{t} H,
$$

since in this case

$$
\left.L_{Y_{t}} \omega_{t}=d\left(Y_{t}\right\rfloor \omega_{t}\right)=d\left(g_{t} H\right)=g_{t} d H \quad \bmod (H) .
$$

Since $(H)$ is the Martinet ideal of $\left(\omega_{t}\right)$, we have

$$
\omega_{t} \wedge\left(d \omega_{t}\right)^{k}=S_{t} H \Omega
$$

for a family $S_{t}$ of nonvanishing functions which has the same regularity in $t$ as in $\omega_{t}$ (this follows from the regularity of the left-hand side and the fact that division by $H$ is a continuous linear operator in the space of smooth functions, see Theorem B1 in Appendix B). Let us show that (7.6) holds for the family $Y_{t}$ defined by

$$
\left.Y_{t}\right\rfloor \Omega=\frac{g_{t}}{S_{t}}\left(d \omega_{t}\right)^{k}
$$

In fact, applying $\left.Y_{t}\right\rfloor$ to $(7.8)$, we get $\left.g_{t}\left(Y_{t}\right\rfloor d \omega_{t}\right) \wedge\left(d \omega_{t}\right)^{k-1}=0$. This relation implies $\left.Y_{t}\right\rfloor d \omega_{t}=0$ (at points where $g_{t}(p)=0$ we have $Y_{t}(p)=0$, and at other points we can use Lemma 4.2 with $\left.\lambda=\left(Y_{t}\right\rfloor d \omega_{t}\right)$ and the fact that contact points are dense). We have shown the first equality in (7.6). In order to prove the second one we apply $\left.Y_{t}\right\rfloor$ to $(7.7)$ and, using (7.8), we obtain that $\left.\left(Y_{t}\right\rfloor \omega_{t}-g_{t} H\right) \cdot\left(d \omega_{t}\right)^{k}=0$. This implies that $\left.Y_{t}\right\rfloor \omega_{t}-g_{t} H=0$ at points where the form $\left(d \omega_{t}\right)^{k}$ does not vanish, in particular, at points where $\omega_{t}$ is contact. By Proposition 3.2, (ii) the set of such points is everywhere dense; therefore $\left.Y_{t}\right\rfloor \omega_{t}-g_{t} H=0$ everywhere, and so (7.6) holds. Proposition 7.1 is proved.

Proof of Proposition 7.2. By Lemma 4.2, (i) and the fact that the set of contact points is dense in $M^{n}$ (Proposition 3.2, (ii)), the equation (7.3) reduces to the following two equations:

$$
\begin{gathered}
\left(L_{Z_{t}} \omega_{t}\right) \wedge \omega_{t} \wedge\left(d \omega_{t}\right)^{k-1}=\mu_{t} \wedge \omega_{t} \wedge\left(d \omega_{t}\right)^{k-1}, \\
\left(L_{Z_{t}} \omega_{t}+h_{t} \omega_{t}\right) \wedge\left(d \omega_{t}\right)^{k}=\mu_{t} \wedge\left(d \omega_{t}\right)^{k}
\end{gathered}
$$

(with unknown $Z_{t}$ and $h_{t}$ ), obtained from (7.3) by external multiplication by the forms $\omega_{t} \wedge\left(d \omega_{t}\right)^{k-1}$ and $\left(d \omega_{t}\right)^{k}$, respectively. 
To solve equation (7.9) we use assumption (7.2). By this assumption

$$
\mu_{t} \wedge \omega_{t} \wedge\left(d \omega_{t}\right)^{k-1}=H \nu_{t}
$$

for some family $\nu_{t}$ of $2 k$-forms, regular in $t$ by Theorem B1 in Appendix B. This permits us to find an explicit solution $Z_{t}$ of (7.9). Namely, since $H$ is a generator of the Martinet ideal of $\left(\omega_{t}\right)$, we have relation (7.7), i.e., $\omega_{t} \wedge\left(d \omega_{t}\right)^{k}=H S_{t} \Omega$, where $S_{t}$ is a family of nonvanishing functions, regular in $t$. Let us show that the family of vector fields $Z_{t}$ defined by the relation

$$
\left.Z_{t}\right\rfloor \Omega=\frac{k}{S_{t}} \nu_{t}
$$

is a solution of the equation (7.9). Relation (7.11) and the fact that $H$ is not a zero divisor imply that $\nu_{t} \wedge \omega_{t}=0$. This and (7.12) imply that

$$
\left.Z_{t}\right\rfloor \omega_{t}=0 .
$$

Consequently, $\left.L_{Z_{t}} \omega_{t}=Z_{t}\right\rfloor d \omega_{t}$ and

$$
\left.\left.\left(L_{Z_{t}} \omega_{t}\right) \wedge \omega_{t} \wedge\left(d \omega_{t}\right)^{k-1}=\left(Z_{t}\right\rfloor d \omega_{t}\right) \wedge \omega_{t} \wedge\left(d \omega_{t}\right)^{k-1}=\frac{1}{k} Z_{t}\right\rfloor\left(\omega_{t} \wedge\left(d \omega_{t}\right)^{k}\right) .
$$

Now (7.9) follows from equalities (7.7) and (7.11)-(7.13).

To prove Proposition 7.2 it remains to solve equation (7.10) with respect to $h_{t}$. Since $\left.Z_{t}\right\rfloor \omega_{t}=0$, then $\left.\left(L_{Z_{t}} \omega_{t}\right) \wedge\left(d \omega_{t}\right)^{k}=(k+1)^{-1} Z_{t}\right\rfloor\left(d \omega_{t}\right)^{k+1}=0$, and the equation (7.10) takes the form

$$
h_{t} \omega_{t} \wedge\left(d \omega_{t}\right)^{k}=\mu_{t} \wedge\left(d \omega_{t}\right)^{k} .
$$

Due to relation (7.7), to prove that this equation has a solution $h_{t}$ it suffices to prove that $\mu_{t} \wedge\left(d \omega_{t}\right)^{k}=H C_{t} \Omega$, where $C_{t}$ is a family of functions, regular in $t$. We shall first prove that

$$
\left(\mu_{t} \wedge\left(d \omega_{t}\right)^{k}\right)(p)=0, \quad \text { for } p \in S .
$$

This follows from relation (7.2). Namely, since $\omega_{t}$ is not contact at a point $p \in S$, thus (7.14) follows from (7.2) by Lemma 4.3, provided that $\omega_{t}(p) \neq 0$. Since the set of points of $S$ at which $\omega_{t}$ vanishes is a subset of the set $\operatorname{Sing}(L)$, the set of points $p \in S$ where $\omega_{t}(p) \neq 0$ is dense in $S$ by Proposition 3.2, (iii). Therefore (7.14) holds at all points $p \in S$. By the property of zeros of the ideal $(H)$ we obtain $\mu_{t} \wedge\left(d \omega_{t}\right)^{k}=H C_{t} \Omega$. Since $\mu_{t}$ and $\omega_{t}$ are regular in $t$, we deduce from Theorem B1 in Appendix B that $C_{t}$ is regular in $t$. Proposition 7.2 is proved.

We have completed the proof of Theorem 1.3. Note that the extension property of $S$ was used only when referring to Proposition 3.1.b., and therefore it is not needed if $\omega_{t}$ is polynomial in $t$ (cf. the remark after Theorem 1.3).

\section{Proof of Theorem 1.4}

Since the Martinet hypersurfaces for $P_{0}=\left(\omega_{0}\right)$ and $P_{1}=\left(\omega_{1}\right)$ are the same and the Martinet ideals have the property of zeros, they are equal and we can choose a common generator $H$ which will be used throughout the proofs. The following proposition holds under the assumptions of Theorem 1.4 and will enable us to reduce the problem to Theorem 1.3. 
Proposition 8.1. Assume that

$$
\omega_{1} \wedge\left(d \omega_{1}\right)^{k-1} \wedge d H=\omega_{0} \wedge\left(d \omega_{0}\right)^{k-1} \wedge d H \quad \bmod (H) .
$$

Then, for the path $\omega_{t}=(1-t) \omega_{0}+t \omega_{1}$ we have

$$
\omega_{t} \wedge\left(d \omega_{t}\right)^{k-1} \wedge d H=B_{t} \omega_{0} \wedge\left(d \omega_{0}\right)^{k-1} \wedge d H \quad \bmod (H),
$$

and

$$
\omega_{t} \wedge\left(d \omega_{t}\right)^{k}=C_{t} \omega_{0} \wedge\left(d \omega_{0}\right)^{k},
$$

where $B_{t}$ and $C_{t}$ are families of functions, polynomial in $t$.

Proof of Theorem 1.4. Let $X_{0}$ and $X_{1}$ be characteristic vector fields of $P_{0}$ and $P_{1}$ defined via the same volume form and the same generator $H$ of the Martinet ideal. Since $L\left(\omega_{1}\right)=L\left(\omega_{0}\right)$, then $X_{1} \wedge X_{0}=0 \bmod (H)$. From condition (A) and the division property in Proposition 3.1.a we obtain $X_{1}=R X_{0} \bmod (H)$ or, equivalently,

$$
\omega_{1} \wedge\left(d \omega_{1}\right)^{k-1} \wedge d H=R \omega_{0} \wedge\left(d \omega_{0}\right)^{k-1} \wedge d H \quad \bmod (H),
$$

where $R$ is a smooth or analytic function. We will later show, using closeness of $\omega_{1}$ to $\omega_{0}$, that $R$ is positive valued. Therefore, we can change the generator of $P_{1}$ for

$$
\hat{\omega}_{1}=R^{1 / k} \omega_{1},
$$

and we get

$$
\hat{\omega}_{1} \wedge\left(d \hat{\omega}_{1}\right)^{k-1} \wedge d H=\omega_{0} \wedge\left(d \omega_{0}\right)^{k-1} \wedge d H \quad \bmod (H) .
$$

Let

$$
\omega_{t}=(1-t) \omega_{0}+t \hat{\omega}_{1}
$$

To prove Theorem 1.4 it is enough to show that the family of Pfaff equations $\left(\omega_{t}\right)$ satisfies the assumptions of Theorem 1.3. Note that we do not need the extension property of $S$, since the family $\omega_{t}$ in (8.6) is polynomial (in fact, affine) in $t$, and in this case Theorem 1.3 was proved without using this assumption.

The equality (8.5) enables us to use Proposition 8.1. It is clear that relations (8.1) and (8.2) imply that the family $\left(\omega_{t}\right)$ satisfies the assumptions (a), (b) and (c) of Theorem 1.3 provided that the functions $B_{t}$ do not vanish on $S$ (then the characteristic line field does not change) and $C_{t}$ do not vanish on $M^{n}$ (then the Martinet ideal does not change), for $t \in[0,1]$.

The fact that $B_{t}$ and $C_{t}$ do not vanish follows from the $C^{\infty}$-closeness of $\omega_{1}$ to $\omega_{0}$ and Theorem B1 in Appendix B. Since $\omega_{1}$ is $C^{\infty}$-close to $\omega_{0}$, then the vector field $X_{1}$ is $C^{\infty}$ close to $X_{0}$. The relation $X_{1}=R X_{0} \bmod (H)$ and continuity of the inverse to the operator $L_{X, H}$ in Theorem B1 imply that there exists a function $\tilde{R}$ that is $C^{\infty}$-close to 1 and equal to $R$ at any point of the Martinet hypersurface $S$. By the property of zeros of the Martinet ideal, $\tilde{R}=R \bmod (H)$. We may replace $R$ by $\tilde{R}$ in (8.3) and in the definition (8.4) of $\hat{\omega}_{1}$. Then $\omega_{t}, t \in[0,1]$, is $C^{\infty}$-close to $\omega_{0}$. Define a family of characteristic vector fields $X_{t}$ by the relation $\left.X_{t}\right\rfloor \Omega=\omega_{t} \wedge\left(d \omega_{t}\right)^{k-1} \wedge d H$. Then $X_{t}, t \in[0,1]$, is $C^{\infty}$-close to $X_{0}$. The equality (8.1) is equivalent to $X_{t}=B_{t} X_{0} \bmod (H)$. We again use continuity of the inverse to the operator $L_{X, H}$ in Theorem B1. By this theorem there exists a function $\tilde{B}_{t}$ that is $C^{\infty}$-close to 1 and equal to $B_{t}$ at any point of $S$. Therefore $B_{t}>0$ at any point of $S$. 
To prove that $C_{t}$ vanishes at no points of $M^{n}$, we also use the $C^{\infty}$-closeness of $\omega_{t}, t \in[0,1]$, to $\omega_{0}$ shown above. Let $\omega_{t} \wedge\left(d \omega_{t}\right)^{k}=Q_{t} H \Omega$, where $\Omega$ is a volume form. Relation (8.2) implies that $Q_{t}=C_{t} Q_{0}$. The $C^{\infty}$-closeness of $\omega_{t}$ to $\omega_{0}$ implies the $C^{\infty}$-closeness of the function $H Q_{t}$ to $H Q_{0}$. By continuity of the inverse to the operator $f \rightarrow f H$ (Theorem B1) we get the $C^{\infty}$-closeness of $Q_{t}$ to $Q_{0}$. Since $Q_{0}$ is a nonvanishing function, then $C_{t}$ is $C^{\infty}$-close to 1 . The proof of Theorem 1.4 is complete.

Proof of Proposition 8.1. It is enough to prove the equalities

$$
\begin{gathered}
\left.X_{0}\right\rfloor\left(\omega_{t} \wedge\left(d \omega_{t}\right)^{k-1} \wedge d H\right)=0 \bmod (H), \\
\left.X_{0}\right\rfloor\left(\omega_{t} \wedge\left(d \omega_{t}\right)^{k}\right)=0 \bmod (H) .
\end{gathered}
$$

Namely, equality (8.7) and condition (A) allow us to use the division properties in Proposition 3.1.b to conclude that $\left.\omega_{t} \wedge\left(d \omega_{t}\right)^{k-1} \wedge d H=B_{t} X_{0}\right\rfloor \Omega \bmod (H)$, and so the validity of relation $(8.1)$, where $B_{t}$ is a family of functions, polynomial in $t$. Equality (8.8) implies that $\omega_{t} \wedge\left(d \omega_{t}\right)^{k}$ vanishes at those points of $S$ at which $X_{0}$ does not vanish. By Proposition 3.2, (iii) the set of such points is dense in $S$; therefore $\omega_{t} \wedge\left(d \omega_{t}\right)^{k}$ vanishes at all points of $S$. By the property of zeros of the Martinet ideal we have $\omega_{t} \wedge\left(d \omega_{t}\right)^{k}=0 \bmod (H)$, and consequently (8.2) holds for some family of functions $C_{t}$, polynomial in $t$.

In order to prove (8.7) and (8.8) we use the assumption of Proposition 8.1 and choose characteristic vector fields $X_{0}$ and $X_{1}$ of $\left(\omega_{0}\right)$ and $\left(\omega_{1}\right)$ equal modulo $(H)$. By Lemma 4.6 we have

$$
\left.\left.X_{0}\right\rfloor \omega_{i}=0 \bmod (H), \quad X_{0}\right\rfloor d H=0 \bmod (H),
$$

for $i=0,1$, and therefore $\left.X_{0}\right\rfloor \omega_{t}=0 \bmod (H)$. It follows that in order to prove (8.7) and (8.8) it suffices to prove the equality

$$
\left.\left(X_{0}\right\rfloor d \omega_{t}\right) \wedge \omega_{t}=0 \quad \bmod (H) .
$$

Due to the property of zeros of the Martinet ideal, it suffices to prove this equality for any point $p \in S$ such that $X_{0}(p) \neq 0$. At such points the 1 -form $d H$ does not vanish and $S$ is smooth. Since $X_{1}=X_{0} \bmod (H)$, using Lemma 4.6 we obtain

$$
\left.\left.\left(X_{0}\right\rfloor d \omega_{0}\right) \wedge \omega_{0}=\left(X_{0}\right\rfloor d \omega_{1}\right) \wedge \omega_{1}=0
$$

in a neighbourhood of $p$ in $S$. Since $X_{0}(p) \neq 0$, then $\omega_{0}(p) \neq 0$ and $\omega_{1}(p) \neq 0$, and therefore these relations imply the equalities

$$
\left.\left.X_{0}\right\rfloor d \omega_{0}=h_{0} \omega_{0}, \quad X_{0}\right\rfloor d \omega_{1}=h_{1} \omega_{1},
$$

which hold in a neighbourhood $U$ of $p$ in $S$. Here $h_{0}$ and $h_{1}$ are functions defined in this neighbourhood. We will show that $h_{0}=h_{1}$; then (8.10) implies that $\left.X_{0}\right\rfloor d \omega_{t}=$ $h_{0} \omega_{t}$, and (8.9) holds in the neighbourhood $U$.

To prove that $h_{0}=h_{1}$ on $U \subset S$, we restrict the relation assumed in Proposition 8.1 to the tangent bundle of $U$. We obtain $\left.\left(\omega_{1} \wedge d \omega_{1}\right)\right|_{U}=\left.\left(\omega_{0} \wedge\left(d \omega_{0}\right)^{k-1}\right)\right|_{U}$ and take the Lie derivative of this relation along the restriction $\left.X_{0}\right|_{S}$ of $X_{0}$ to $S$ (recall that $X_{0}$ is tangent to $S$ ). As in the proof of Proposition 6.1, we obtain the required equality $h_{0}=h_{1}$. Proposition 8.1 is proved. 


\section{Appendix A. Division properties}

In this Appendix we present a general theorem on division properties of the exterior (respectively, interior) product with a section $X$ of a vector bundle. This theorem is proved in [DJ] and implies our Propositions 3.1.a and 3.1.b. Our results hold in the categories $C^{s}$, where $s=\infty$ or $s=\omega$.

Let $M$ be a paracompact differential manifold. Consider a vector bundle $E$ over $M$ of rank $m$ and denote by $E^{*}$ its dual bundle. Let $\Lambda_{r}=\Lambda_{r}(E)$ denote the $r$ th exterior power of $E, r=0,1, \ldots, m$, with $\Lambda_{0}=M \times \mathbb{R}$ and $\Lambda_{1}=E$. We denote by $\Lambda_{r}(M ; E)$ the linear space of sections of $\Lambda_{r}$ (smooth or real analytic, depending on the category).

Any section $\alpha$ of $E$ defines the linear operator of exterior multiplication by $\alpha$, which gives the complex

$$
0 \rightarrow \Lambda_{0}(M) \rightarrow \Lambda_{1}(M ; E) \rightarrow \cdots \cdots \rightarrow \Lambda_{m}(M ; E),
$$

with the operator $\partial_{\alpha}=\partial_{\alpha}^{p}: \Lambda_{p}(M ; E) \rightarrow \Lambda_{p+1}(M ; E)$ defined by $\partial_{\alpha}^{p}(\gamma):=\alpha \wedge \gamma$.

Consider a section $X$ of the dual bundle $E^{*}$. This section defines the operator of the interior product with $X, X\rfloor: \Lambda_{r}(M ; E) \rightarrow \Lambda_{r-1}(M ; E)$. Given a local basis $e_{1}, \ldots, e_{m}$ of $E$, the operator of the interior product with $X$ is defined on the elements of a local basis of $\Lambda_{r}$ by

$$
X\rfloor\left(e_{i_{1}} \wedge \cdots \wedge e_{i_{m}}\right)=\sum_{j=1}^{m}(-1)^{j-1}\left\langle X, e_{i_{j}}\right\rangle e_{i_{1}} \wedge \cdots \breve{e}_{i_{j}} \cdots \wedge e_{i_{m}},
$$

where $\breve{e}_{i_{j}}$ means absence of $e_{i_{j}}$ and $\langle\cdot, \cdot\rangle$ denotes the duality product between $E^{*}$ and $E$. Clearly, $(X\rfloor)^{2}=0$; so the operator $\left.X\right\rfloor$ defines the complex

$$
0 \rightarrow \Lambda_{m}(M ; E) \rightarrow \Lambda_{m-1}(M ; E) \rightarrow \cdots \rightarrow \Lambda_{1}(M ; E) \rightarrow \Lambda_{0}(M ; E) .
$$

Let $S$ be a closed subset of $M$. Denote by $\Lambda_{r}(M, S ; E) \subset \Lambda_{r}(M ; E)$ the subspace of sections of $\Lambda_{r}(M ; E)$ vanishing at all points of $S$, and let

$$
\Lambda_{r}(S ; E)=\Lambda_{r}(M ; E) / \Lambda_{r}(M, S ; E)
$$

denote the quotient space.

Any element $\alpha$ of $\Lambda_{1}(S ; E)$ defines the unique operator

$$
\partial_{\alpha}^{p}: \Lambda_{p}(S ; E) \rightarrow \Lambda_{p+1}(S ; E)
$$

(the quotient of the operator of exterior multiplication), which gives the complex

$$
0 \rightarrow \Lambda_{0}(S) \rightarrow \Lambda_{1}(S ; E) \rightarrow \cdots \cdots \rightarrow \Lambda_{m}(S ; E) .
$$

Given a section $X$ of $E^{*}$, the operator $\left.X\right\rfloor$ defines the following complex on the quotient spaces:

$$
0 \rightarrow \Lambda_{m}(S ; E) \rightarrow \Lambda_{m-1}(S ; E) \rightarrow \cdots \rightarrow \Lambda_{1}(S ; E) \rightarrow \Lambda_{0}(S ; E) .
$$

We define the invariant $d_{p}(X)=\operatorname{depth}\left(I_{p}\right)$, where $I_{p}$ is the ideal of function germs at $p \in M$ generated by the coefficients $a_{1}, \ldots, a_{m}$ of $X$ in a local basis of $E^{*}$ (cf. Definition 1.2 in Section 1). Similarly, given a pair $(H, X)$ of a function $H$ and a section $X$ of $E^{*}$ on $M$, we define $d_{p}(H, X)$ as the maximal length of a regular sequence of function germs that begins with the germ $H_{p}$ of $H$ at $p$ and has further elements in $I_{p}$ (cf. Definition 1.4). Analogously we define the invariants $d_{p}(\alpha)$ and $d_{p}(H, \alpha)$. 
Statements (i) and (ii) of the following theorem hold in the $C^{\infty}$ and $C^{\omega}$ categories, for $0 \leq q \leq n-1$.

Theorem A. (i) If $\alpha$ satisfies the condition $d_{p}(\alpha) \geq q+1$ for all $p \in M$ such that $\alpha(p)=0$, then the complex $(A .1)$ is exact up to $\Lambda_{q}(M ; E)$. Similarly, if $d_{p}(X) \geq q+1$ for all $p \in M$ such that $X(p)=0$, then the complex (A.2) is exact up to $\Lambda_{m-q}(M ; E)$.

(ii) Let $H$ be a function on $M$ such that the ideal $(H)$ has the property of zeros, and let $S=\{H=0\}$. If $\alpha$ is a section of $E$ on $M$ such that $(H, \alpha)$ satisfies $d_{p}(H, \alpha) \geq q+2$ for all $p \in S$ such that $\alpha(p)=0$, then the complex (A.3) is exact up to $\Lambda_{q}(S ; E)$. Similarly, if $X$ is a section of $E^{*}$ on $M$ and $d_{p}(H, X) \geq q+2$ for all $p \in S$ such that $X(p)=0$, then the complex (A.4) is exact up to $\Lambda_{m-q}(S ; E)$.

(iii) If the assumptions of (i) hold, then, in the $C^{\infty}$ category, the complex (A.1) splits up to $\Lambda_{q-1}(M ; E)$ and the complex (A.2) splits up to $\Lambda_{m-q+1}(M ; E)$. If the assumptions of (ii) hold and $S$ has the extension property, then the complex (A.3) splits up to $\Lambda_{q-1}(S ; E)$ and the complex $(A .4)$ splits up to $\Lambda_{m-q+1}(S ; E)$. Here the corresponding spaces are equipped with the $C^{\infty}$ topology and are considered as Fréchet spaces (quotient Fréchet spaces).

Above, a complex $0 \rightarrow L_{m} \rightarrow \cdots \rightarrow L_{m-q+1} \rightarrow L_{m-q} \rightarrow \cdots$ defined by the operators $\partial_{i}: L_{i} \rightarrow L_{i-1}$ is called exact up to $L_{m-q}$ if $\operatorname{Im} \partial_{i+1}=\operatorname{ker} \partial_{i}$ for $i=$ $m, m-1, \ldots, m-q$, and it splits up to $L_{m-q+1}$ if the $L_{i}$ are linear topological spaces, $\operatorname{Im} \partial_{i}$ are closed subspaces of $L_{i-1}$ and each $\partial_{i}: L_{i} \rightarrow L_{i-1}$ has a continuous right inverse $K_{i}$ defined on $\operatorname{Im} \partial_{i}$, for all $i=m, m-1, \ldots, m-q+1$.

The above theorem follows from Theorems 2.1 and 2.2 in [DJ]. In the local case (of germs) statements (i) and (ii) follow from a well-known algebraic result on exactness of the Koszul complex, cf. e.g. [E] or JZh2, Appendix 1.

Proof of Proposition 3.1.a. In the even-dimensional case the first implication follows trivially from statement (i) in Theorem A if we take the bundle $E$ equal to the cotangent bundle $E=T^{*} M$, the dual $E^{*}=T M$, and consider the complex (A.2). $\left(\Lambda_{r}(M, E)\right.$ is identified with the space of differential $r$-forms on $M$.) The second implication follows analogously from the same statement by taking $E=T M$ and the complex (A.1).

In the odd-dimensional case the first implication follows in a similar way from statement (ii) in Theorem A concerning the complex (A.4). This is because the property of zeros of $(H)$ allows us to identify the elements of $\Lambda_{r}(S ; E)$ with the equivalence classes of differential $r$-forms modulo $(H)$ (cf. our convention on notation $\bmod (H)$ presented after Definition 1.3). The second implication follows analogously from statement (ii) in Theorem A concerning the complex (A.3).

Proof of Proposition 3.1.b. The existence of $\mu_{t}$ and $f_{t}$ for any fixed $t$ follows from Proposition 3.1.a. We have to show the regularity of these families in $t$. We shall prove the regularity of $\mu_{t}$ (the proof of regularity of $f_{t}$ is analogous). If $\nu_{t}$ depends on $t$ polynomially, then Proposition 3.1 allows us to construct $\mu_{t}$ polynomial in $t$, and the regularity follows trivially. In the general case our arguments are different for the categories $C^{\omega}$ and $C^{\infty}$.

In the $C^{\omega}$ category we use the following fact: if a sequence $a_{1}, \ldots, a_{r}$ of real analytic function germs at $p \in M$ is regular in the ring of analytic function germs at $p$, then it is regular when considered as a sequence in the ring of real analytic function germs of the variables $(x, t) \in M \times \mathbb{R}$ at $\left(p, t_{0}\right)$, for any $t_{0} \in[0,1]$. Using 
local coordinates, this fact can be easily proved for the case of formal power series using the definition of regular sequence in the ring of formal power series of the variables $x_{1}, \ldots, x_{n}, t$. Then, using the fact that the ring of formal power series is faithfully flat over the ring of convergent series (see Malgrange Mlg, Chapter 3 ), we see that it also holds for converging series and so for germs of analytic functions. Using the above fact we see that the assumption (A) holds over the manifold $\tilde{M}=M \times I$, where $I$ is an open interval containing $[0,1]$ on which the analytic family $\nu_{t}$ is well defined by analytic extension. Thus we can use Theorem A over the manifold $\tilde{M}$, i.e., for the bundles $E=T^{*} M$ and $E^{*}=T M$ pulled back to $\tilde{M}$ by the canonical projection $M \times I \rightarrow M$.

In the $C^{\infty}$ category, in the even-dimensional case the smooth dependence of $\mu_{t}$ on $t$ follows from statement (iii) in Theorem A. By this statement there exists a continuous right inverse operator $K$ to the linear operator $X\rfloor: \Lambda_{r+1}(M ; E) \rightarrow$ $\Lambda_{r}(M ; E)$, for $r=n-1$ and $r=n-2$, and we can define $\mu_{t}=K \nu_{t}$. Here $E=T^{*} M$ and $\Lambda_{r}(M ; E)=\Lambda_{r}(M)$, the space of differential $r$-forms on $M$.

In the $C^{\infty}$ category, in the odd-dimensional case we also use statement (iii) of Theorem A and the extension property of $S$. Namely, for $E=T^{*} M$ we define $\mu_{t}=\left.\lambda K \nu_{t}\right|_{S}$, where $K: \Lambda_{n-1}(S ; E) \rightarrow \Lambda_{n}(S ; E)$ is the continuous right inverse operator to $X\rfloor: \Lambda_{n}(S ; E) \rightarrow \Lambda_{n-1}(S ; E)$, and $\lambda: \Lambda_{n}(S ; E) \rightarrow \Lambda_{n}(M ; E)$ is a continuous linear operator of extension.

\section{Appendix B. Continuity of Division}

Continuity of division in the cases presented below is needed in the main proofs and will be proved separately. Let $C^{\infty}(M)$ and $V e c t^{\infty}(M)$ be the spaces of smooth functions and smooth vector fields on $M$, with the $C^{\infty}$ topology. Let $C^{\infty}(M, S)$ and $V e c t^{\infty}(M, S)$ be the subspaces of functions (vector fields) on $M$ vanishing on the Martinet hypersurface $S \subset M$. The quotient Fréchet spaces

$$
C^{\infty}(S)=C^{\infty}(M) / C^{\infty}(M, S), \quad \operatorname{Vect}^{\infty}(S ; T M)=\operatorname{Vect}^{\infty}(M) / V e c t^{\infty}(M, S)
$$

can be identified with the space of smooth functions on $S$ and the space of smooth sections of the tangent bundle $T M$ restricted to $S$, respectively.

Given a Pfaff equation on $M^{2 k}$ and a characteristic vector field $X$, we consider the linear operator

$$
L_{X}: C^{\infty}(M) \rightarrow \operatorname{Vect}^{\infty}(M), \quad L_{X}(f)=f X .
$$

For a Pfaff equation on $M^{2 k+1}$, a characteristic vector field $X$ and a generator $H$ of the Martinet ideal we consider the linear operators

$$
L_{H}: C^{\infty}(M) \rightarrow C^{\infty}(M), \quad L_{H}(f)=H f
$$

and

$$
L_{X, H}: C^{\infty}(S) \rightarrow \operatorname{Vect}^{\infty}(S ; T M), \quad L_{X, H}([f])=[f X],
$$

where $[\cdot]$ denotes the equivalence class in the corresponding quotient space.

Theorem B1. If the characteristic vector field $X$ satisfies condition (A) and in the odd-dimensional case the Martinet ideal $(H)$ has the property of zeros, then each of the linear operators $L_{X}, L_{H}$ and $L_{X, H}$ is injective, has closed image, and has continuous inverse defined on the image. 
Proof. By the Banach open mapping theorem in Fréchet spaces it suffices to prove that each of the operators $L_{X}, L_{H}$, and $L_{X, H}$ is injective and has closed image.

The injectivity of the operators $L_{X}, L_{H}$ and $L_{X, H}$ follows from Proposition 3.2, (i), (ii), (iii), respectively. The closedness of the image of the operator $L_{H}$ follows from the global property of zeros implied by Definition 1.5 - by this property the image of $L_{H}$ coincides with the closed subspace $C^{\infty}(M, S) \subset C^{\infty}(M)$ of functions vanishing on $S$.

To prove the closedness of the image of the operators $L_{X}$ and $L_{X, H}$, we use Proposition 3.1. By Proposition 3.1, (i) the image of $L_{X}$ coincides with the kernel of the continuous operator $V e c t^{\infty}(M) \rightarrow \Gamma^{\infty}\left(\Lambda^{2} T M\right)$ defined by $Y \rightarrow X \wedge Y$, where $\Gamma^{\infty}\left(\Lambda^{2} T M\right)$ is the space of smooth sections of the skew-symmetric product of the tangent bundle $T M$, with the $C^{\infty}$ topology. Similarly, by Proposition 3.1, (ii) the image of $L_{X, H}$ coincides with the kernel of the continuous operator $V e c t^{\infty}(S) \rightarrow$ $\Gamma^{\infty}\left(\Lambda^{2}(S ; T M)\right)$ given by $[Y] \rightarrow[X \wedge Y]$, where $\Gamma^{\infty}\left(\Lambda^{2}(S ; T M)\right)$ is the space of smooth sections over $S$ of the skew-symmetric product of the tangent bundle $T M$ (with the $C^{\infty}$ topology) and [ $\left.\cdot\right]$ denotes the equivalence class in the corresponding quotient space. The kernel of this operator is a closed subspace of $V e c t^{\infty}(S ; T M)=$ $V e c t^{\infty}(M) / V e c t^{\infty}(M, S)$. The proof is complete.

It is natural to ask if it is possible to replace the $C^{\infty}$-closeness of $\omega_{1}$ to $\omega_{0}$ in Theorems 1.2 and 1.4 by $C^{r}$-closeness with some $r$. Any attempt at answering this question requires modification of Theorem B1, which was used in the proofs of Theorems 1.2 and 1.4. Proving Theorem 1.2, we had to show that the function $A_{t}, t \in[0,1]$, does not vanish at any point of $M$. In the proof of Theorem 1.4 we had to show that the functions $B_{t}, t \in[0,1]$, do not vanish at points of $S$ and the functions $C_{t}, t \in[0,1]$, do not vanish at points of $M$. The $C^{\infty}$-closeness of $\omega_{1}$ to $\omega_{0}$ given as an assumption in Theorems 1.2 and 1.4 and the continuity of the inverse to the operators $L_{X}, L_{H}$ and $L_{X, H}$ allowed us to obtain the $C^{\infty}$-closeness of $A_{t}$, $B_{t}$ and $C_{t}$ to 1 . Of course, to conclude that these functions do not vanish, their $C^{0}$-closeness to 1 would be enough.

We introduce the following topological characteristic of a linear injective operator $L: C^{\infty}(M) \rightarrow C^{\infty}(M)$ or $L: C^{\infty}(M) \rightarrow V e c t^{\infty}(M)$ or $L: C^{\infty}(S) \rightarrow \operatorname{Vect}^{\infty}(S)$. Denote by $m \in\{0,1,2, \ldots ; \infty\}$ the minimal $m$ such that for any $s \geq 0$ the convergence to 0 of the sequence of sections $L\left(f_{n}\right)$ in the $C^{s+m}$ topology implies the convergence to 0 of the sequence of functions $f_{n}$ in the $C^{s}$ topology. This means that the inverse to $L$ behaves not worse than a linear differential operator of order $m$. Note that by Theorem B1 we have $m\left(L_{X}\right), m\left(L_{H}\right)$, and $m\left(L_{X, H}\right) \leq \infty$.

In many cases the numbers $m\left(L_{X}\right), m\left(L_{H}\right)$ and $m\left(L_{X, H}\right)$ are finite and can be found or estimated from above, see examples below. Tracing the construction of the functions $A_{t}, B_{t}$ and $C_{t}$ in the proofs of Theorems 1.2 and 1.4, it is easy to check that if these numbers are finite, then:

1. the $C^{0}$-closeness of $A_{t}$ to 1 holds provided that the 1 -form $\omega_{1}$ is close to $\omega_{0}$ in the $C^{r}$ topology with $r=2 m\left(L_{X}\right)+2$;

2. the $C^{0}$-closeness of $B_{t}$ to 1 holds provided that the 1 -form $\omega_{1}$ is close to $\omega_{0}$ in the $C^{r}$ topology with $r=2 m\left(L_{X, H}\right)+2$;

3. the $C^{0}$-closeness of $C_{t}$ to 1 holds provided that the 1 -form $\omega_{1}$ is close to $\omega_{0}$ in the $C^{r}$ topology with $r=m\left(L_{X, H}\right)+m\left(L_{H}\right)+2$.

Therefore in Theorems 1.2 and 1.4 the $C^{\infty}$-closeness of $\omega_{1}$ to $\omega_{0}$ can be replaced by the closeness in a weaker topology, and we obtain the following result. 
Theorem B2. In Theorem 1.2 the $C^{\infty}$-closeness of $\omega_{1}$ to $\omega_{0}$ can be replaced by the $C^{r}$-closeness with $r=2 m\left(L_{X}\right)+2$. In Theorem 1.4 the $C^{\infty}$-closeness of $\omega_{1}$ to $\omega_{0}$ can be replaced by the $C^{r}$-closeness with

$$
r=\max \left(2 m\left(L_{X, H}\right)+2, m\left(L_{X, H}\right)+m\left(L_{H}\right)+2\right) .
$$

Examples $(n=2 k)$. 1. If $X$ has no singular points, then it is clear that $m\left(L_{X}\right)=$ 0 . Therefore the $C^{\infty}$-closeness of $\omega_{1}$ to $\omega_{0}$ in Theorem 1.2 can be replaced by $C^{2}$-closeness. We obtain Theorem 0.1.

2. If the 1-jet of $X$ vanishes at no points of the manifold, then it is easy to prove that $m\left(L_{X}\right) \leq 1$. Therefore the $C^{\infty}$-closeness of $\omega_{1}$ to $\omega_{0}$ in Theorem 1.2 can be replaced by $C^{4}$-closeness.

Examples $(n=2 k+1)$. 1. If $\left(\omega_{0}\right)$ is a Martinet distribution, i.e., $d H(p) \neq 0$ and $X(p) \neq 0$ for any $p \in S=\{H=0\}$, then it is easy to prove that $m\left(L_{H}\right) \leq 1$ and $m\left(L_{X, H}\right)=0$. Therefore the $C^{\infty}$-closeness of $\omega_{1}$ to $\omega_{0}$ in Theorem 1.4 can be replaced by $C^{3}$-closeness. We obtain Theorem 0.2 .

2. Assume that $d H(p) \neq 0$ for any $p \in S=\{H=0\}$. Then the restriction of $X$ to $S$ is a smooth vector field $\left.X\right|_{S}$ on $S$. Assume that the 1-jet of $X_{S}$ does not vanish. In this case $m\left(L_{H}\right) \leq 1$ and $m\left(L_{X, H}\right) \leq 1$. Therefore the $C^{\infty}$-closeness of $\omega_{1}$ to $\omega_{0}$ in Theorem 1.4 can be replaced by $C^{4}$-closeness.

\section{ACKNOWLEDGMENTS}

While working on this paper we have profited from discussions with several colleagues. We are especially thankful for helpful advice obtained from Paweł Domański, Jean-Paul Gauthier, Pierre Milman and Richard Montgomery.

\section{REFERENCES}

[A] A. Agrachev, Methods of Control Theory in Nonholonomic Geometry, Proc. Internat. Congress of Math., Zürich 1994, Vol. 2, pp. 1473-1483, Birkhäuser, Basel, 1995. MR 97f:58051

[ArIl] V. I. Arnold and Yu. S. Ilyashenko, Ordinary Differential Equations, in Encyclopaedia of Math. Sci. Vol. 1, Dynamical Systems 1, Springer-Verlag (1986). MR 87e:34049] MR 89g:58060

[BS] E. Bierstone and G. W. Schwarz, Continuous linear division and extension of $C^{\infty}$ functions, Duke Math. Journal 50 (1983), 233-271. MR 86b:32010

[BH] R. L. Bryant and L. Hsu, Rigidity of integral curves of rank 2 distributions, Inventiones Math. 114 (1993), 435-461. MR 94j:58003

[C] H. Cartan, Variétés analytiques réelles et variétés analytiques complexes, Bull. Soc. Math. de France 85 (1957), 77-99. MR 20:1339

[DJ] P. Domański and B. Jakubczyk, Linear continuous division for exterior and interior products (accepted to Proc. Amer. Math. Soc.).

[E] D. Eisenbud, Commutative Algebra, Springer-Verlag, New York 1994. MR 97a:13001

[Gol] A. Golubev, On the global stability of maximally nonholonomic two-plane fields in four dimensions, Internat. Math. Res. Notices, 11, 523 - 529, 1997. MR 98g:57043

[G] J. W. Gray, Some global properties of contact structures, Annals of Math. 69, (1959), 421-450. MR 22:3016

[JP] B. Jakubczyk and F. Przytycki, On J. Martinet's conjecture, Bull. Polish Acad. Sci. Math. 27 (1979), No. 9, 731-735. MR 82e:58003

[JZh1] B. Jakubczyk and M. Zhitomirskii, Odd-dimensional Pfaffian equations; reduction to the hypersurface of singular points, Comptes Rendus Acad. Sci. Paris, Série I t. 325 (1997), 423-428. MR 99e:58003

[JZh2] B. Jakubczyk and M. Zhitomirskii, Local reduction theorems and invariants for singular contact structures, Ann. Inst. Fourier, 51 (2001), 237-295. MR 2002c:58001 
[LS] W. Liu and H. Sussmann, Shortest paths for sub-Riemannian metrics on rank-two distributions, Mem. Amer. Math. Soc., Vol. 118 (1995), No. 564. MR 96c:53061

[Mlg] B. Malgrange, Ideals of differentiable functions, Oxford University Press, 1966. MR 35:3446

[Mar] J. Martinet, Sur les singularités des formes différentielles, Ann. Inst. Fourier, Vol. 20, No.1 (1970), 95-178. MR 44:3333

[Mon] R. Montgomery, A survey on singular curves in sub-Riemannian geometry, J. Dynamical and Control Systems, Vol.1, No.1 (1995), 49-90. MR 95m:53060

[MZh] R. Montgomery and M. Zhitomirskii, Geometric approach to Goursat flags, Annales de l'Institut Henri Poincaré, Analyse Nonlineaire, Vol. 18, No. 4 (2001), 459-493. MR 2002d:58004

[Ru] J. M. Ruiz, The Basic Theory of Power Series, Advanced Lectures in Mathematics, Vieveg, Wiesbaden, 1993. MR 94i:13012

[TEG] C. B. Thomas, Y. Eliashberg, and E. Giroux, 3-dimensional contact geometry, in "Contact and Symplectic Geometry", Publ. Newton Institute 8, Cambridge University Press, Cambridge, 1996, 48-65. MR 98b:53026

[Zh1] M. Zhitomirskii, Singularities and normal forms of odd-dimensional Pfaff equations. Functional. Anal. Appl., Vol. 23, (1989), No. 1, pp. 59-61. MR 90i:58007

[Zh2] M. Zhitomirskii, Typical singularities of differential 1-forms and Pfaffian equations, Translations of Math. Monographs, Vol. 113, Amer. Math. Soc., Providence, RI, 1992. MR 94j:58004

Institute of Mathematics, Polish Academy of Sciences, Śniadeckich 8, 00-950 Warsaw, Poland and Institute of Applied Mathematics, University of Warsaw, Poland

E-mail address: B.Jakubczyk@impan.gov.pl

Department of Mathematics, Technion, 32000 Haifa, Israel

E-mail address: mzhi@techunix.technion.ac.il 